\title{
Electroconvulsive Seizure Behavior in Drosophila: Analysis of the Physiological Repertoire Underlying a Stereotyped Action Pattern in Bang-Sensitive Mutants
}

\author{
Jisue Lee and Chun-Fang Wu \\ Department of Biological Sciences, University of lowa, lowa City, lowa 52242
}

\begin{abstract}
Drosophila bang-sensitive mutants display a remarkable stereotyped behavioral sequence during mechanical disturbances. This seizure repertoire consists of initial and delayed bouts of spasm interposed with paralysis and followed by recovery of activity and a period of refractoriness to further stimulation. Electroconvulsive stimuli across the brain induced a similar seizure behavior in tethered flies, in which corresponding electrophysiological events could be readily recorded in indirect flight muscles [dorsal longitudinal muscles (DLMs)] of the giant fiber (GF) pathway. The DLM physiological repertoire consisted of initial and delayed discharges (IDs and DDs), a response failure and recovery, followed by a refractory period. Interestingly, wild-type flies also displayed the same electroconvulsive repertoire, albeit inducible only at higher stimulus intensities and with briefer expression. The DLM repertoire presumably originated from activities of distinct neural circuits subserving normal function and reflected the general sequence
\end{abstract}

of excitation and depression of the nervous system as a whole, as shown by simultaneous recordings along the different body axes. The well characterized GF pathway facilitated localization of circuits responsible for response failure and ID and DD motor patterns by surgical manipulations, recording-stimulating site analysis, and genetic mosaic studies. A flight pattern generator is most likely the major contributor to shaping the DD pattern, with modifications by active integration of individual motor neurons and associated interneurons. The robust electroconvulsive repertoire of DLMs provides a convenient window for further genetic analysis of the interacting neural mechanisms underlying a stereotyped action pattern in Drosophila, which shows striking parallels with aspects of seizure in mammalian species.

Key words: electroconvulsive spike discharge; seizure susceptibility; refractory period; gynandromorph mosaics; giant fiber pathway; bas bss
Mutations of several genes in Drosophila lead to a distinct seizure behavior during mechanical stress. These mutants, collectively known as the bang-sensitive mutants (Benzer, 1971; Ganetzky and $\mathrm{Wu}, 1982$ ), including bang sensitive (bas) (Grigliatti et al., 1973) and bang senseless (bss) (Jan and Jan, 1978; Ganetzky and $\mathrm{Wu}, 1982$ ), provide interesting model systems for research into the neural basis of a stereotyped seizure behavior. The characteristic spasm-and-paralysis sequence (Benzer, 1971; Burg and $\mathrm{Wu}, 1987$ ) is followed by a refractory period (Ganetzky and $\mathrm{Wu}$, 1982), during which the mutant flies are no longer sensitive to mechanical disturbances.

Such bang-sensitive behavior displays interesting parallels with certain forms of mammalian epilepsy and seizure evoked by electrical shock or pharmacological agents. These aspects include a stereotyped action pattern (Fisher, 1989) and a period of refractoriness to further stimulus (Nutt et al., 1981). Epileptic seizures result from defects of considerable molecular and genetic heterogeneity (Noebels, 1996; McNamara, 1999; Stafstrom and Tempel, 2000) and have not been understood within a framework of a single unified mechanism. Drosophila is amenable to incisive

\footnotetext{
Received July 1, 2002; revised Oct. 8, 2002; accepted Oct. 9, 2002.

The work was supported by National Institutes of Health Grants NS18500 and NS28528. We thank Dr. Jeff Engel for his help in this study, Dr. Martin Burg for making the double mutants bas $^{1} b_{s s^{1}}$ and $e a s^{2}$ bas $^{1}$, and Dr. Wilfried Janning for providing a ring-X gynandromorph stock. We also thank Dr. Jeff Engel, Dr. Sudipta Sarawati, and Brett Berke for comments on an early version of this manuscript.

Correspondence should be addressed to Dr. Chun-Fang Wu, Department of Biological Sciences, University of Iowa, Iowa City, IA 52240. E-mail: cfwu@blue.weeg.uiowa.edu.

Copyright (C) 2002 Society for Neuroscience $0270-6474 / 02 / 2211065-15 \$ 15.00 / 0$
}

genetic analyses, and thus the study of bang-sensitive mutants may reveal principles across phyla that underlie stability of neural network functioning and aberrant synchronicity of massive, abnormal activities.

An important step in the study of bang-sensitive seizure mechanisms is to establish physiological correlates in an identified neural pathway. The neural elements in the cervical giant fiber (GF) reflex pathway have been identified anatomically (King and Wyman, 1980; Ikeda and Koenig, 1988) and characterized physiologically (Tanouye and Wyman, 1980; Engel and Wu, 1992; Trimarchi and Schneiderman, 1993, 1996). Here, we demonstrate an electroconvulsion-induced seizure repertoire in tethered flies, composed of an initial spasm, paralysis, delayed spasm, activity recovery, and seizure refractoriness, similar to that induced by mechanical stress in unrestrained bang-sensitive mutants. Simultaneous video and electrophysiological recordings in dorsal longitudinal muscles of the GF pathway established a stereotyped physiological sequence that correlated with the entire electroconvulsion-induced behavioral repertoire. The physiological repertoire consisted of initial discharge (ID), response failure $(\mathrm{F})$, delayed discharge (DD), response recovery (R), and a refractory period. Significantly, the same repertoire was also inducible electroconvulsively in wild-type flies, although at higher stimulus intensities and with a shorter duration of expression, indicating recruitment of neural circuits subserving normal function. Thus, we asked how this dorsal longitudinal muscle (DLM) signature sequence reflects activities of the CNS, how the individual events in the repertoire differ in their properties, and how they depend on the cellular elements in the GF pathway for 
expression. We found that removal of GABAergic inhibition by picrotoxin feeding induced a rhythmic bursting activity distinct from the electroconvulsive repertoire, reminiscent of two types of seizure patterns induced by electrical stimulus trains and GABAergic blockade in mammalian seizure models. We further examined the roles of candidate neural circuits that drive normal activity patterns in shaping DLM electroconvulsive discharges. Multiple-site stimulation and recording, surgical manipulations, and genetic mosaic analysis (Hotta and Benzer, 1972) confirmed that identifiable circuit elements in separate anatomical loci are responsible for the various components of the seizure repertoire.

Preliminary accounts of this work have appeared previously in abstract form (Lee and Wu, 1998, 2000; Wu and Lee, 2001).

\section{MATERIALS AND METHODS}

\section{Fly strains}

Bang-sensitive mutants in Drosophila melanogaster that were studied in detail include bang sensitive (bas: bas ${ }^{1}$, bas ${ }^{2}$ ) (Grigliatti et al., 1973), bang senseless (bss: bss ${ }^{1}$, bss $^{2}$ ) (Jan and Jan, 1978; Ganetzky and Wu, 1982), and the double mutant, bas $^{1}$ bss $^{1}$ (Burg and Wu, 1987). The two different alleles of bas and bss yielded similar results in behavioral and physiological experiments, and pooled data for each gene were shown in quantitative analyses (see Figs. 4, 6). The wild-type strain used was CantonSpecial (CS). Other bang-sensitive mutants that were also examined in this study include easily shocked (eas: eas ${ }^{1}$, eas ${ }^{2}$ ) (Ganetzky and $\mathrm{Wu}$, 1982), knockdown ( $k d n: k d n^{1}$ ) (Ganetzky and Wu, 1982), slam dance (sda: $s d a^{i s o 61.10}$, sda $a^{i s o 7.8}$ ) (Pavlidis and Tanouye, 1995), and technical knockout (tko: tko ${ }^{25 t}$ ) (Judd et al., 1972). Before experiments, we confirmed the extreme seizure behavior of these lines as described previously (Burg, 1987) to ensure that the phenotypes were not masked by accumulation of unidentified modifiers. We also examined another mutant 2206 that shows impaired locomotion after mechanical stress (Schubiger et al., 1994). Bang sensitivity of mutant flies was tested (cf. Ganetzky and Wu, 1982) with a vortex mixer (K-550-G, Scientific Industries, Bohemia, NY) at the maximum speed setting ( $55 \mathrm{~Hz}$ vibration at an amplitude of $5 \mathrm{~cm}$ ) as specified previously. Some of the mutant alleles were kept on chromosomes carrying visible markers and/or in balanced stocks by using balancer chromosomes $\left(b^{1} s^{1} / \mathrm{Y} / \mathrm{X}\right.$ X $y f, g^{2} b a s^{2} f / \mathrm{Y} / \mathrm{X} \mathrm{X}$ y $f, g b_{s s}^{1}$, cho sn $b s s^{2}, g b_{a} s^{1} s d b s s^{1} / \mathrm{Y} / \mathrm{X} \mathrm{X}$ y $f, e^{e a s} s^{1} / \mathrm{Y} / \mathrm{X} \mathrm{X}, k d n^{1} v$ $f / \mathrm{Y} / \mathrm{X} \mathrm{X} y f$, and $t k o^{25 t} / \mathrm{Y} / \mathrm{X} \mathrm{X}$ ) [see Lindsley and Zimm (1992) for details of the morphological markers and special chromosomal aberrations]. Flies were reared at room temperature $\left(21-24^{\circ} \mathrm{C}\right)$ and kept in $9.5 \times 2.5 \mathrm{~cm}$ glass vials with standard cornmeal medium. Adult flies used in the experiments were $3-10 \mathrm{~d}$ old.

\section{Behavior recordings}

Electroconvulsive seizure behavior induced by $200 \mathrm{~Hz}$ electroconvulsive stimuli $(50 \mathrm{~V}, 0.1 \mathrm{msec}$ and $2 \mathrm{sec}$ train duration) was recorded on videotape simultaneously with electrophysiological recording using tethered bang-sensitive mutant flies (see below). The mechanical stressinduced seizure behavior in unrestrained mutant flies was also videotaped after vortexing. Images were captured by a CCD camera (Hitachi Solid State color camera, $\mathrm{kp}-\mathrm{C} 100 \mathrm{U}$ ) attached to a trinocular dissecting microscope and recorded by a videocassette recorder (Sony SLV-696 HF). The analog image was converted into digital files (Video Vision, Radius Inc., San Jose, CA) using the software Videof usion (version 1.6.1, Videofusion Inc.) and Adobe Photoshop (version 5.0, Adobe system).

\section{Drug feeding}

Wild-type CS flies were examined for the effects of blocking inhibitory synaptic transmission. Flies were allowed to feed on $1 \%$ agarose medium containing $1 \mathrm{mg} / \mathrm{ml}$ brilliant blue, $0.1 \mathrm{M}$ sucrose, and $2 \mathrm{~mm}$ picrotoxin. The control medium was identical in composition except for the drug. Ingestion of the food medium was evident as indicated by the food-dye color in the gut visible through the cuticle. Flies were exposed to the testing medium between 30 and 60 min, until a substantial amount of food was ingested.

\section{Electrophysiological methods}

Physiological recordings were mostly performed on the GF pathway responsible for the jump-and-flight escape response (Tanouye and

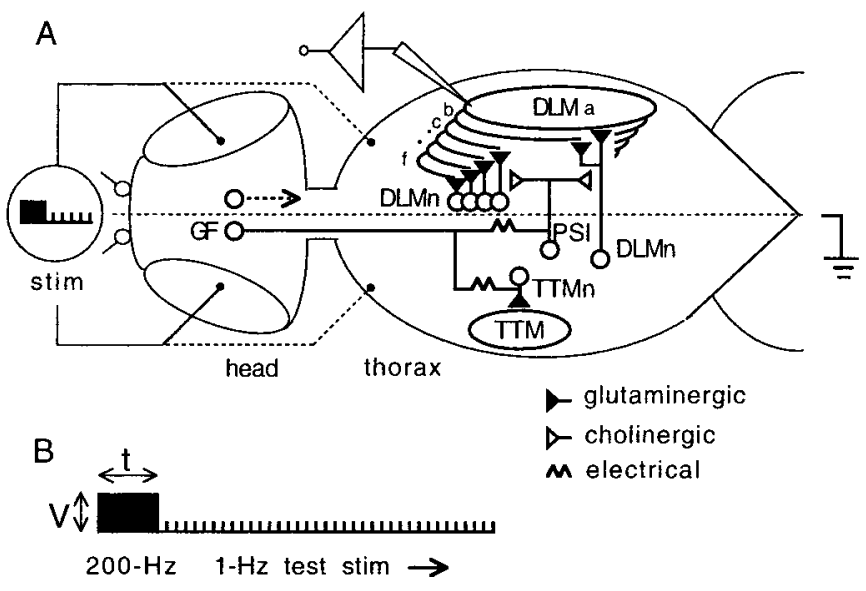

Figure 1. The giant fiber $(G F)$ pathway and stimulation protocol for electroconvulsive seizure. $A$, Electrical stimuli were delivered across the brain or thorax, and physiological responses were recorded at the various outputs of the GF pathway. One of the bilaterally symmetrical GF pathway pairs, which is responsible for jump-and-flight escape reflex, is schematized. The GF neuron in the brain activates a TTMn (jump) directly but recruits DLMns (flight) via the PSI interneuron in the thorax. Three different types of identified synapses ( glutaminergic, cholinergic, electrical) are indicated. GF, Giant fiber; PSI, peripherally synapsing interneuron, $D L M a-f$, a stack of six dorsal longitudinal muscle fibers; $D L M n$, DLM motor neuron; TTM, tergotrochanteral muscle; TTMn, TTM motor neuron. $B$, Electroconvulsive stimulation protocol. Electroconvulsive stimuli $(200 \mathrm{~Hz}$ of $0.1 \mathrm{msec}$ pulses $)$ were followed by test pulses ( $1 \mathrm{~Hz}$ of $0.1 \mathrm{msec}$ pulses) to detect transmission failure in the GF pathway during seizure. Stimulus strength of the $200 \mathrm{~Hz}$ stimulus train could vary in voltage $(V)$ or duration $(t)$.

Wyman, 1980; Wyman and Thomas, 1983; Trimarchi and Schneiderman, 1993) in tethered flies. Methods for muscle recording in the GF pathway (Fig. 1A) have been described (Engel and Wu, 1992, 1996, 1998). Flies were etherized briefly and glued to a metal wire between the neck and thorax using nail polish (Gorczyca and Hall, 1984). After recovery for at least $30 \mathrm{~min}$, a high-frequency electroconvulsion stimulus train $(200 \mathrm{~Hz}$, $0.1 \mathrm{msec}$ pulses) (Fig. $1 B$ ) was used to induce seizure. Tungsten electrodes (uninsulated) were used for stimulation and recording. Responses were picked up with an AC preamplifier (WPI DAM-5A, New Haven CT) (filter bandwidth from $0.1 \mathrm{~Hz}$ to $>30 \mathrm{kHz}$, reference grounded) and recorded with pulse code modulation (Neuro Data DR-384, New York, $\mathrm{NY}$ ) on videotape at a sampling rate of $44 \mathrm{kHz}$.

The frequency of the electroconvulsive stimuli $(200 \mathrm{~Hz})$ approaches the GF after frequency with 50\% response in wild type (Engel and $\mathrm{Wu}$, 1992). The voltage-duration relationship of the stimulus train for seizure induction (compare Fig. 4C) was established for each genotype by varying voltage from 10 to $100 \mathrm{~V}$ in $10 \mathrm{~V}$ increments in combination with $0.1,0.5,1.0$, or $2.0 \mathrm{sec}$ train durations. This comprehensive characterization provided a basis for the adequate ranges of stimulus voltage and train duration selected for the experiments shown in other figures. After the $200 \mathrm{~Hz}$ electroconvulsive stimulus (manual switching time of $\sim 3 \mathrm{sec}$ ), test pulses of $0.1 \mathrm{msec}$ and $24 \mathrm{~V}$ (or higher voltage that is sufficient to evoke short-latency responses) (cf. Engel and Wu, 1996) were delivered at $1 \mathrm{~Hz}($ Fig. $1 B)$ to examine the response failure and recovery of the GF pathway. An interval of at least $10 \mathrm{~min}$ was allowed between presentations of electroconvulsive stimuli to avoid the effect of refractoriness. The $200 \mathrm{~Hz}$ electrical stimulation could be delivered repeatedly as many as 40 times in a wild-type fly and $>20$ for bas or bss mutants, yielding reproducible seizure responses.

Giant fiber pathway. A schematic drawing of the GF pathway is shown in Figure $1 A$ (see Figs. 11, 12, insets). For clarity, only one GF pathway of the bilateral pair is presented. The GF receives inputs from various sources, including the visual and olfactory systems (Trimarchi and Scheiderman, 1995), and projects its output to the thoracic ganglion forming several synapses for jump and flight responses (King and Wyman, 1980; Ikeda and Koenig, 1988). The GF forms mixed electrical and chemical synapses (Blagburn et al., 1999) with a peripherally synapsing interneuron (PSI), which in turn projects via cholinergic synapses 
(Gorczyca and Hall, 1984) to each of the five glutaminergic motor neurons that innervate six contralateral DLMs, stacking from dorsal to ventral (Fig. 1 $A, a-f$ ). The somata of four motor neurons (DLMns), innervating DLMs $c-f$, are located contralaterally to the GF-PSI chain. However, DLMs $a$ and $b$ are innervated by a single motor neuron, receiving input from the same GF-PSI, with the motor neuron soma located on the ipsilateral side. On the other hand, the same GF directly forms an electrical synapse (King and Wyman, 1980; Baird et al., 1993) with the ipsilateral tergotrochanteral muscle motor neuron (TTMn), which forms a glutaminergic synapse with the TTM on the same side.

Stimulation and recording loci. The electrodes were positioned as follows: a pair of stimulating electrodes into the eyes, recording electrodes into specific flight (DLM) and/or jump (TTM) muscles, and a reference electrode into the abdomen (Fig. 1A). Placement of recording electrodes into identified muscles (different DLMs and the TTM) of the GF pathway was guided by cuticular markers (Levine and Hughes, 1973) and characteristic latency and shape of action potentials (Tanouye and Wyman, 1980; Engel and Wu, 1992, 1996). Recording sites were also confirmed using dye staining through the insertion sites (cf. Engel and $\mathrm{Wu}, 1992)$. To examine the requirement of the head for initiating a physiological repertoire, electroconvulsive stimulation was delivered directly to the anterior portion of the thorax, bypassing the head in an intact or a decapitated fly (compare Fig. 10). The wound in decapitated flies was sealed with wax to prevent dehydration.

Two-channel simultaneous recordings were made in the left and right pairs of DLMs and TTMs or in DLM pairs on the same side (compare Fig. 11). General activity of the nervous system was recorded between an electrode positioned in the retina and the reference electrode in the abdomen. In addition, activity trafficking between the head and thorax was recorded by inserting an electrode into the cervical connectives (compare Fig. 5). Unlike other experiments, flies were positioned ventral side up for the cervical recording. With the fly turned over, a right-angled electrode was used to record corresponding DLM activities. Its insertion in the DLM was guided with a mirror and confirmed by action potential properties. All physiological experiments were performed at room temperature $\left(22-27^{\circ} \mathrm{C}\right)$.

\section{Genetic mosaic flies}

Gynandromorph mosaic flies composed of hemizygous ( bas $^{1}$ bss $\left.^{1} / \mathrm{O}\right)$ and heterozygous $\left(\right.$ bas $^{1}$ bss $^{1} /++$ ) tissues were constructed by crossing $y w$ $s p l /$ ring-X (or $y w l z /$ ring-X) virgin females with $g b_{a s}{ }^{1} S D b s s^{1} / Y$ males [see Lindsley and Zimm (1992) for the visible markers and the ring-X chromosome, $\left.\operatorname{In}(1) w^{v C}\right]$. The random loss of the unstable ring- $\mathrm{X}$ chromosome (containing normal copies of the bas and bss genes), during development of the female offspring ( $g$ bas ${ }^{1} S D b_{s s}{ }^{1} /$ ring-X), creates hemizygous patches surrounded by the heterozygous tissue $\left(\right.$ bas $^{1}{ }^{1} s^{1} / \mathrm{O} / /$ $b a \mathrm{~s}^{1}$ bss $^{1} /++$ ) (cf. Hotta and Benzer, 1972). Hemizygous tissue expressing the bas $^{1}$ bss ${ }^{1}$ phenotype could be identified by morphology of male characteristics (sex comb, smaller wing size, abdominal banding pattern, and genitalia) or surface markers [garnet eye $(g)$ and scalloped wing $(S D)]$. Bilateral mosaic flies as indicated by the above surface markers were selected for electrophysiological recordings (compare Fig. 12). Stocks with unstable ring- $X$ for generating mosaics at a high rate were maintained by selecting female flies with visible mosaic patches $(y w$ $\mathrm{spl} / \mathrm{ring}-\mathrm{X} / / \mathrm{y} w \mathrm{spl} / \mathrm{O})$ in each generation to cross with males ( $y w \mathrm{spl} / \mathrm{Y}$ $B^{s+}$ ) for propagation. We studied bas bss double-mutant mosaics, which displayed striking physiological differences between hemizygous (mutant) and heterozygous (control) tissues, consistent with clear distinctions of behavioral phenotypes between hemizygous (male) and heterozygous (female) double mutants (cf. Burg, 1987). Physiological recording also demonstrated distinct seizure responses between heterozygous (female; data not shown) and hemizygous (male) non-mosaic flies. Additional $g$ bas $^{1}$ sd eas $^{2} f$ double-mutant mosaics were also constructed in this study and produced consistent results (data not shown).

\section{RESULTS}

\section{Drosophila bang-sensitive and electroconvulsive seizure behaviors and their physiological correlates}

Seizure repertoires evoked by mechanical and

electroconvulsive shocks

On mechanical agitation, such as vortexing or tapping the culture vial on the bench, all bang-sensitive mutants (including bas, bss, eas, $k d n$, sda, and $t k o$; see Materials and Methods) showed a stereotyped sequence of initial muscle spasm (uncoordinated movements), paralysis (lack of motion and responsiveness), delayed spasm, and recovery (normal posture). The two spasms were manifested by collapse of the body, high-frequency wing flapping, leg extension, and fully curved abdomen (see Fig. $2 A$ for an example of $b s s$.) Furthermore, female flies often laid eggs after mechanical agitation (Fig. $2 A$, arrowhead). These spasms and paralysis were reproducible with a predictable time to onset of the delayed spasm or to recovery of normal posture in bas and bss flies, which was significantly lengthened in bas bss double-mutant flies (Fig. 2B). This orderly behavioral sequence implies that it results from patterned activities of neural circuits rather than unassociated events in individual muscles. Significantly, wild-type flies (Fig. 2C) and other categories of behavioral mutants do not show such behavioral repertoire even after extreme mechanical stimulation, such as vortexing the culture vial for tens of seconds (see Materials and Methods).

We found that electroconvulsive stimulation (Fig. 1B) (see Materials and Methods) delivered to the brain of a tethered bang-sensitive mutant fly reproducibly induced the repertoire of seizure behavior, which paralleled the bang-sensitive behavioral sequence in unrestrained mutant flies. As confirmed by video recording, the same sequence of initial spasm, paralysis, delayed spasm, and recovery was preserved in the tethered flies after electroconvulsive stimulation (Fig. $3 A$ ). Although mechanically induced seizure is a unique phenotype seen only in bang-sensitive mutants, we found that electroconvulsive seizure could be induced reproducibly in tethered wild-type flies and mutants of other categories at more extreme stimulus intensities (Lee and Wu, 1998, 2000). Thus, it is possible to analyze mutant effects on seizure susceptibility and expression with well defined protocols in tethered flies.

\section{Physiological correlates of the seizure repertoire in indirect flight muscles}

The physiological correlates of the behavioral repertoire could be systematically analyzed in the GF pathway in which the motor outputs of jump and flight muscles coordinate the escape reflex (Fig. 1A) (see Materials and Methods). A physiological repertoire (Fig. $3 B$ ) could be detected in the DLMs that temporally correlated with the entire behavioral sequence after electroconvulsive stimulation. The corresponding sequence in the DLM repertoire was an initial discharge of spikes, response failure, delayed discharge, and response recovery. The failure and recovery of the GF pathway were monitored by recording the DLM response to $1 \mathrm{~Hz}$ test pulses (Figs. $1 B, 3 B$ ). Recovery time or failure duration were defined as the interval between the electroconvulsive stimulus and the restoration of the response in DLM to five consecutive test pulses.

These characteristic behavioral and physiological patterns were observed in all tethered bang-sensitive mutants examined, including bas, bss, eas, kdn, sda, and tko (Fig. 4) (and data not shown). In this report, we focused on the classical bang-sensitive mutants, bas and bss, because they have been well characterized genetically (Ganetzky and Wu, 1982), physiologically (Jan and Jan, 1978; Engel and Wu, 1994; Pavlidis and Tanouye, 1995), and behaviorally (Burg, 1987). Interactions between the two mutations were revealed in bas $^{1}$ bss $^{1}$ double-mutant flies.

Traces of muscle activities recorded intracellularly in the muscle DLMa (Fig. 1 $A$ ) are shown in Figure $4 A$ for the different 
Figure 2. Mechanically induced seizure in the bang-sensitive bas, bss, and bas bss flies. $A$, Seizure repertoire in bss mutants. Mechanical shock (10 sec vortexing; see Materials and Methods) induced a stereotyped behavioral sequence of initial spasm, paralysis, delayed spasm, and recovery of normal posture in both male (top panels) and female (bottom panels) mutant flies. Seizing females often lay eggs (arrowheads). The initial spasm, which occurred during the first few seconds after vortexing, was not captured in videotaping. $B$, Duration of seizure induced by vortexing vials containing five or fewer flies. Onset time of delayed spasm (hatched bar) and time to recovery (open bar) are quantified on the basis of the numbers of flies indicated. $C$, Seizure susceptibility in different genotypes as indicated by percentage of flies displaying the seizure repertoire after vortexing. In this and the following figures, error bars indicate SD. ${ }^{* *} p<0.01 ;{ }^{* * *} p<$ 0.001 .

Figure 3. Electroconvulsively induced seizure behavior and its physiological correlates in the DLM flight muscle of a $b s s^{I}$ fly. $A$, Electroconvulsive stimulation applied to a tethered fly induced a behavioral repertoire of initial spasm, paralysis, delayed spasm, and recovery to normal posture. $B$, The same electroconvulsive stimulation in $A$ induced a physiological repertoire of initial discharge $(I D)$, which occurred during initial spasm, response failure $(F)$, occurring during paralysis, delayed discharge $(D D)$, during delayed spasm, and response recovery $(R)$, concurring with recovery of normal posture in a DLM. In this and the following figures, the filled triangle indicates onset of $\mathrm{DD}$, and the open triangle indicates R (recovery of consecutive responses) in traces of physiological recording.
A
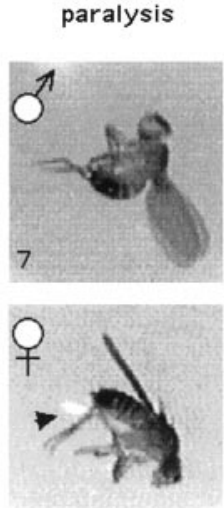

43
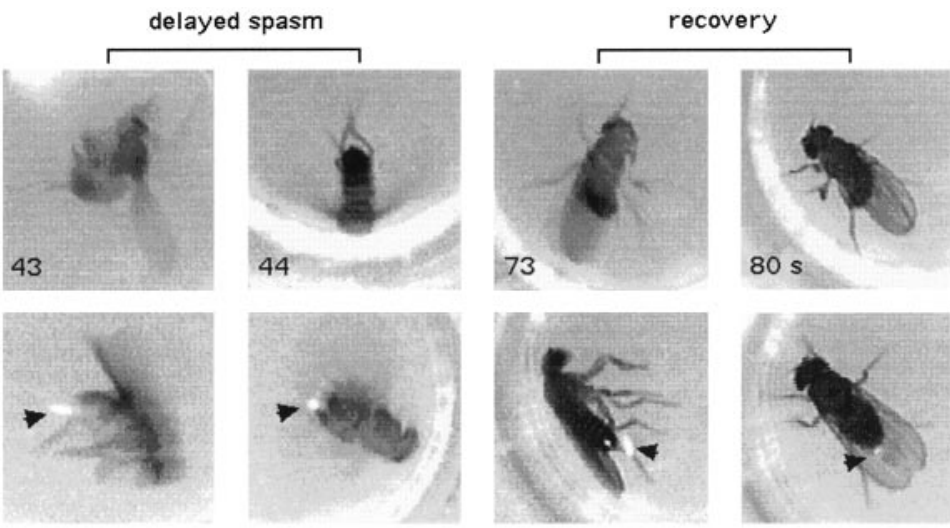

B

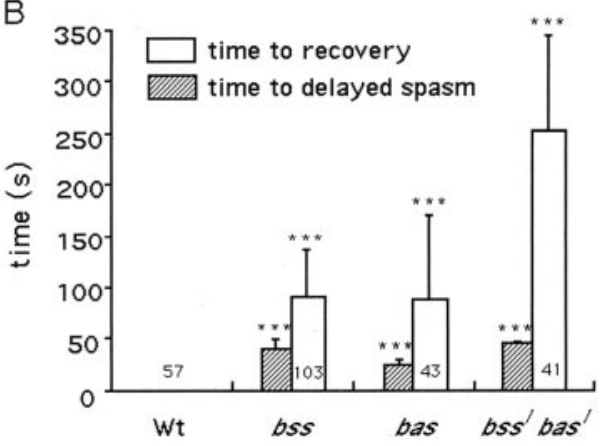

C

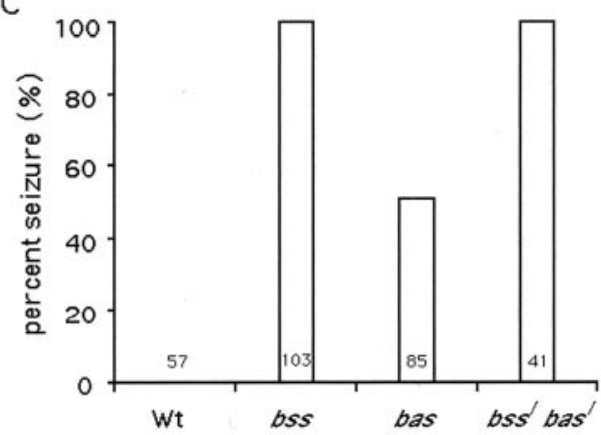

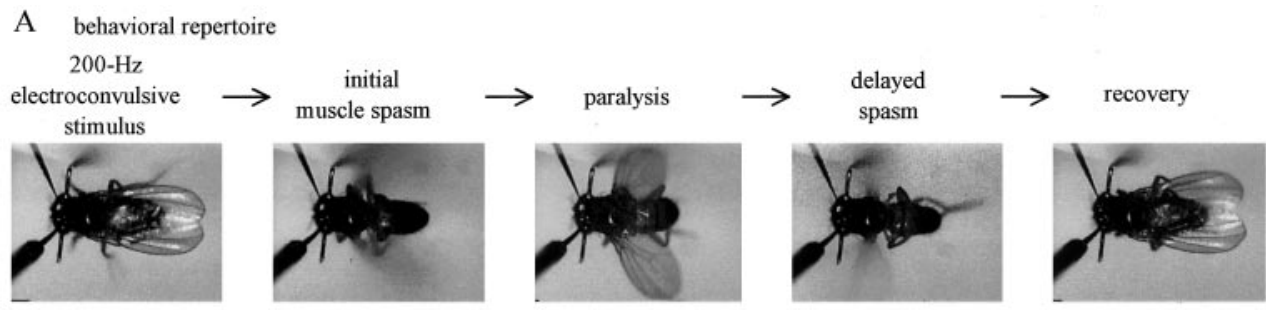

B physiological repertoire $200-\mathrm{Hz} \longrightarrow$ initia electroconvulsive $\rightarrow$ discharge stimulus

(ID)
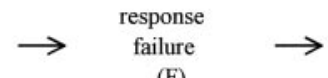

delayed

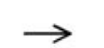

response
recovery

(DD)

(R)

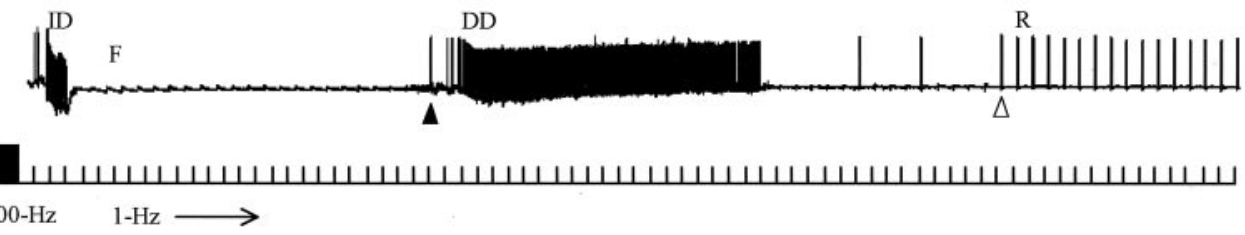

genotypes. (Tungsten electrode recordings in this and following figures produced apparently varying amplitudes of spikes. This was caused by electrode polarization, which is worsened during higher spike frequency and by larger stimulus artifacts.) Temporal parameters such as the onset time and duration of each event were used to characterize the genotype-specific profiles of ID, $\mathrm{DD}$, and $\mathrm{F}$ in wild type, $b a s^{1}, b s s^{1}$, and bas $^{1} b s s^{1}$ based on large samples of flies (Fig. 4B). The above parameters were highly reproducible among flies within each genotype as evidenced by the relatively small SDs in Figure $4 B$. As shown in Figure 4, $A$ and $B$, after ID ended, there was a period of response failure to $1 \mathrm{~Hz}$ brain stimulation, which is shortest in wild type and longest in $b a s^{1} b s s^{1}$. DD occurred during response failure and lasted for tens of seconds, whereas ID subsided within a few seconds (Fig. $4 A, B)$. These observations indicate that DD generation does not rely on the functioning of the GF pathway and that DD and ID may involve separate neural circuits.

We further compared threshold levels for evoking a physiological repertoire for wild-type, bas, bss, and $b a s^{1} b s s^{1}$ flies. Adequate $0.1 \mathrm{msec}, 200 \mathrm{~Hz}$ stimuli were obtained by adjusting either voltage or duration of the stimulus train (Fig. 4C). Threshold was defined as the voltage-duration combinations at which $50 \%$ of examined flies displayed $\mathrm{F}$ and DD in the physiological repertoire. Thus the threshold curves indicate minimally effective stimulus conditions (Fig. 4C) and provide a basis for selecting stimulus voltages and train durations appropriate for each genotype in the experiments 

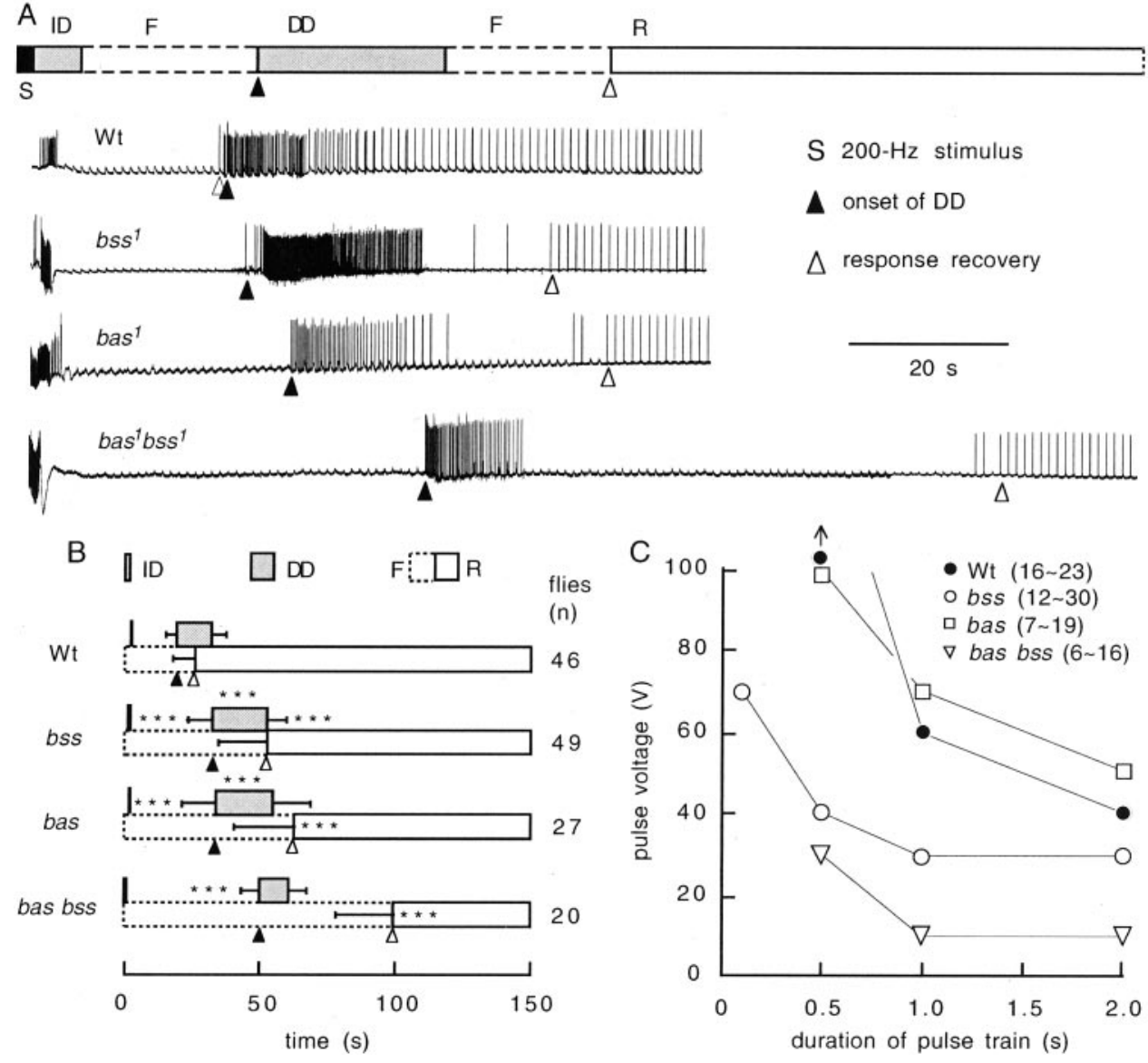

S $200-\mathrm{Hz}$ stimulus

onset of DD

$\triangle$ response recovery

$20 \mathrm{~s}$
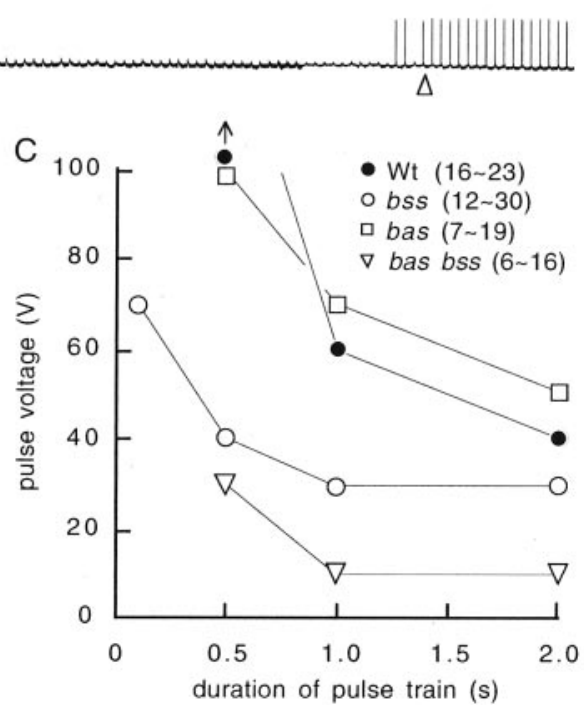

Figure 4. The DLM physiological repertoire and induction threshold in wild-type and bang-sensitive mutants. $A$, Wild type $(W t), b s s^{1}, b a s^{1}$, and $b a s^{1} b s s^{1}$ all showed the same stereotyped sequence of initial discharge $(I D)$, response failure $(F)$, delayed discharge $(D D)$, and recovery $(R)$ in the DLM. Note response failures (indicated by stimulus artifacts of $1 \mathrm{~Hz}$ test pulses) before recovery (open triangles) of the DLM action potentials (see Results). $B$, Profiles of the physiological repertoires in the bangsensitive mutants. ID, DD, and F for each genotype were characterized based on the number of flies indicated. Time periods of DLM response failure $(F)$ and recovery of GF pathway transmission $(R)$ are indicated. For this figure and Figure 6, statistics are pooled from different alleles for single mutants (bas: bas ${ }^{1}$, bas $^{2}$; bss: bss ${ }^{1}, b s s^{2}$ ), and the double-mutant data are based on the bas ${ }^{1}$ $b_{s s}{ }^{l}$ combination. $C$, Duration-voltage relationship of stimulus trains for electroconvulsive seizure induction. Increasing the $200 \mathrm{~Hz}$ pulse train duration lowered the adequate voltage for induction of the DLM repertoire. Data points represent threshold levels of $50 \%$ success. One fly contributed only one test result at each intensity. The ranges of sample sizes for data points of each genotype are indicated. At stimulus duration of $\leq 0.5 \mathrm{sec}$, seizure induction in wild-type flies was not attainable $(C)$, and the voltage required for seizure induction correlated with the sensitivity to mechanical stimulation (compare with Fig. 2C).

to follow. We found that the voltage level required for seizure induction with pulse trains of $0.5 \mathrm{sec}$ or shorter (Fig. $4 C$ ) correlated well with sensitivity to mechanical agitation in free-moving flies of each genotype (Fig. $2 C$ ), i.e., mechanically induced seizure was most readily produced in bas bss but not attainable in wild-type flies. Presumably, electroconvulsion is more effective than mechanical shocks for seizure induction in all genotypes and can provide a more precise and convenient tool to quantify seizure susceptibility.

As compared with bss and $b a s^{1} b s s^{1}$, the higher threshold for bas electroconvulsive seizure (Fig. 4C) was consistent with its lower percentage of paralysis induced mechanically in unrestrained flies (Fig. 2C). Furthermore, in bas $^{1}$ bss $^{1}$ flies, the enhanced susceptibility to electroconvulsion (Fig. $4 C$ ) as well as the increased durations of the evoked repertoire (Fig. 4B) correlated well with its extreme sensitivity to mechanical stress and prolonged paralysis (Fig. 2B). It should be noted that although the same sequence is seen in both electroconvulsively and mechanically induced seizure behavioral repertoires, the electroconvulsive response appeared to be less variable and occurred in a compressed time scale (approximately half) (compare Figs. 2B, 4B).

\section{Expression of the physiological repertoire}

The above results established a close correlation between the DLM physiological repertoire and the seizure behavioral events. This prompted us to examine how this physiological signature sequence detectable in DLMs reflects activities of other portions of the CNS, how the individual components in the sequence differ in their properties, and how this stereotyped action pattern is modified during a second electroconvulsion.
General electroconvulsive activity in the nervous system during the DLM repertoire

The seizure behavioral repertoire described above involves different body parts, which may require concurrent activities in a large number of neural circuits. In addition to the physiological events detected in the DLM of the GF pathway, we examined possible correlations between the DLM repertoire with the general electrical activity and signals trafficking between the brain and thorax (Fig. 5). Differential recording between electrodes positioned in one retina and the abdomen preferentially registered action potentials occurring near the electrodes or propagating between the head and thorax (Fig. 5A,B). There were several distinct features in these recordings. First, a suppression of the general activity level was evident during the period of DLM response failure. Second, large spikes were detected during the DLM burst periods of ID and DD (simultaneous recording traces in Fig. $5 A, B$ ). Third, some spike activities of smaller amplitudes (Fig. 5A, $B$, asterisk) usually preceded the larger spikes, but it is not known whether they were involved in the initiation of the DD motor pattern generation. Therefore, the DLM repertoire primarily reflects the general activity levels of the neuromuscular systems during electroconvulsive seizures. A closer inspection indicated, however, that there was no strict correspondence between the occurrence of these extracellularly recorded spikes with individual DLM spikes (expanded panels $a$ and $b$ for wildtype and $c$ and $d$ for $b s s^{1}$ mutant flies). Furthermore, we recorded action potentials traveling between the head and thorax directly from the cervical connectives after brain stimulation. Although the identity and direction of traffic for individual spikes were not 

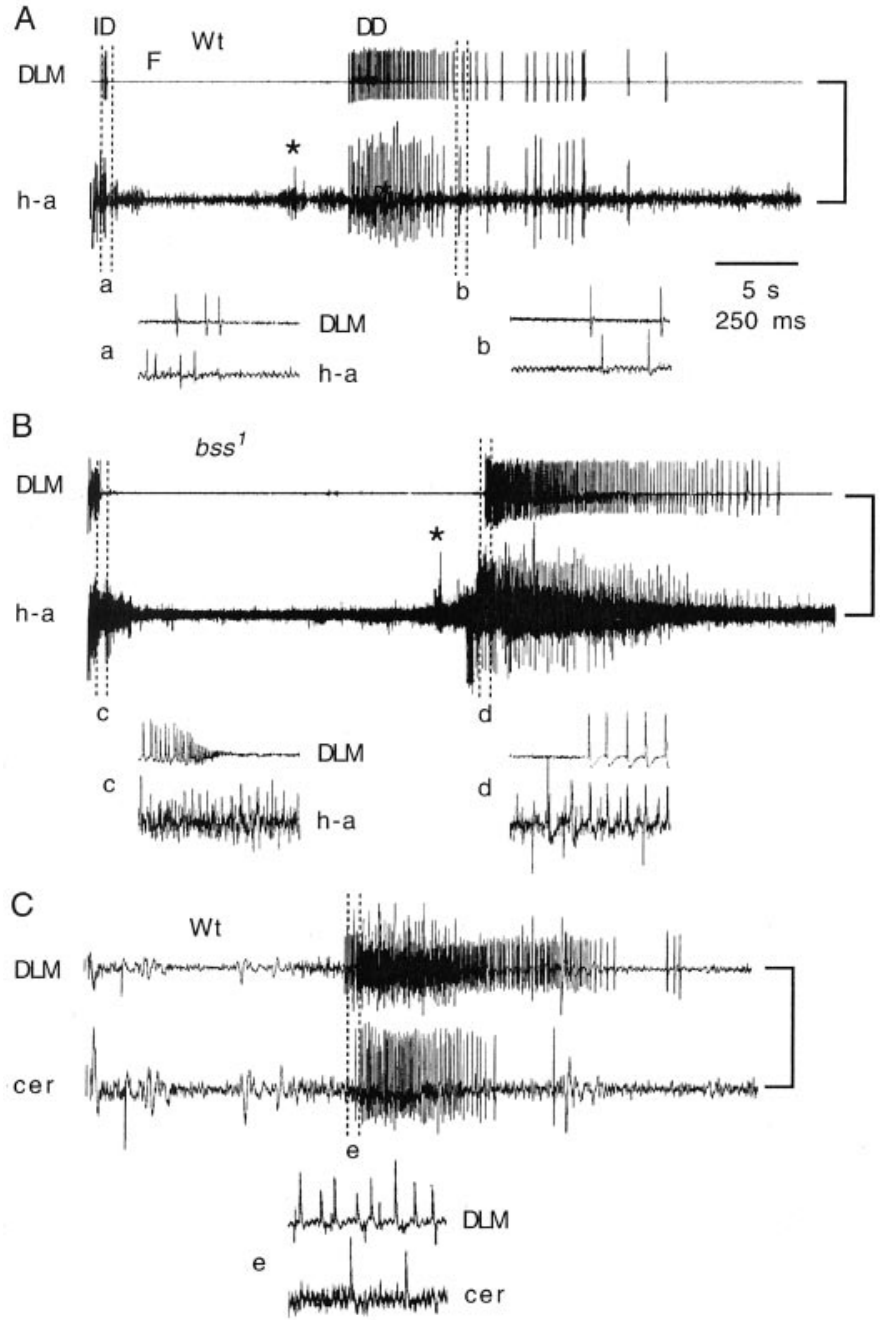

Figure 5. General nerve activities evoked by electroconvulsive brain stimulation in wild-type and $b s s^{1}$ flies. $A, B$, Correlation of DLM activities with differential recording between the electrodes positioned in the head and abdomen in wild-type $(A)$ and $b s s^{1}(B)$ flies. These paired recordings demonstrated activity suppression and bursting events in the CNS that corresponded to DLM response failure $(F)$ and discharges $(I D, D D)$. However, individual spikes in the DLM were not coincidental with those in head-abdomen recordings (segments $a-d$ are expanded $20 \times$ for temporal comparison; $a, b$, for wild-type; $c, d$, for $b s s^{1}$ flies). $C$, Signal trafficking between the head and thorax detected in the cervical connectives correlated with simultaneous DLM recording after an electroconvulsive stimulus in a wild-type fly $(e, 20 \times)$. $D L M$, Muscle recording; $h-a$, differential recording between head and abdomen; cer, cervical connective recording.

resolved, cervical connective recordings again showed activities temporally correlated with DLM activities (Fig. 5C) without a strict one-to-one correspondence between spikes (expanded panel e). These observations demonstrate that general CNS activities with an overall temporal pattern underlie electroconvulsive seizure and that the effects of bang-sensitive mutations are pervasive throughout the CNS. Thus the DLM repertoire provides refined, quantitative indicators of mutational effects and a convenient window for probing CNS activity during seizure.

Distinct stimulus-response relations for ID, $F$, and DD

The DLM seizure repertoire may be generated under the influence of multiple inputs from separable neural circuits, as sug- gested by the temporally ordered ID, F, and DD and by their differences in sensitivity to electroconvulsive stimuli and mutational modification. The DLM responses induced at various stimulus intensity levels (50-100 V, 2 sec duration) in a wild-type fly is shown in Figure $6 A$. F and DD reached a plateau expression level above $70 \mathrm{~V}$. In contrast, ID expression was suppressed beyond a suprathreshold level $(100 \mathrm{~V})$. The distinct nonmonotonic dependence of ID on stimulus intensity was true for all genotypes examined (Fig. 6) (and data not shown). Furthermore, distinctions in expression levels between ID and DD (or F) were paralleled by the probability of seizure induction at different stimulus strengths. By varying duration of the stimulus train (200 $\mathrm{Hz}$ ) at a fixed voltage $(50 \mathrm{~V})$, we determined the induction probability as a function of stimulus strength and observed a general trend of increased percentage of flies displaying $\mathrm{F}$ and DD when the stimulus was lengthened (Fig. $6 C, D$ ). In contrast, prolonged stimulation appeared to exert an inhibitory effect on ID induction (Fig. 6B). Therefore, maximal plateau expression of $\mathrm{F}$ and DD provided robust and convenient indicators for mutant characterization. In contrast, the optimal ID expression required fine-tuning of stimulus intensity, which varied among flies. It is worth noting that a plateau expression of F and DD parallels the phenomenon in mammalian species that stimulus intensities beyond $1.2 \times$ the electroconvulsion threshold result in the same maximal seizure expression (Kupferberg, 2001).

\section{Stereotyped action pattern of the physiological repertoire}

The ability to induce the electroconvulsive seizure even in wildtype flies suggests a natural capacity of neural circuits in Drosophila to exhibit a fixed sequence of activity under extreme stimulus conditions. The seizure repertoire is reminiscent of the release of fixed-action patterns of certain reflexive behaviors (Reichert and Wine, 1982). Usually, such distinct behavioral repertoires proceed to the end once they are initiated, even if additional stimuli were introduced. This property was demonstrated in the Drosophila electroconvulsive repertoire by using a pair of stimuli to determine the extent to which the second stimulus modifies the repertoire in progress. It was observed that before the onset of DD (during GF response failure), the second brain stimulus exerted essentially no influence on the repertoire (Fig. 7). The stimulus delivered after the onset of DD introduced a brief suppression of DD activities and delayed the recovery of the GF pathway response to $1 \mathrm{~Hz}$ test pulses. The extent of modification depended on genotypes, with the least influence in wild type and the greatest in the double mutant (Fig. 7). The lack of significant influence of the second stimulus was observed for a wide range of intensities, even for suprathreshold stimuli shown in Figure 7 (note ID suppression by suprathreshold stimuli; compare Fig. 6).

\section{Refractoriness of the physiological repertoire}

The mild effects of a second stimulus on the physiological repertoire initiated by the previous stimulus prompted an examination of the opposite situation, i.e., how a previous stimulus influences the effectiveness of the ensuing stimulus. The mechanically induced seizure behavior in bang-sensitive mutant flies exhibits a striking refractoriness (Ganetzky and Wu, 1982). For instance, in bas and bss mutant alleles, spasms and paralysis could not be induced within a refractory period of tens of minutes after a previous seizure episode.

To test whether similar refractoriness also exists for the electroconvulsively induced physiological repertoire, paired stimuli 
Figure 6. Electroconvulsion stimulus intensity-response relationship in wild-type and bang-sensitive mutant flies. $A$, Stimulus intensity-dependent expression of the DLM physiological repertoire in a wild-type fly. ID evoked by $200 \mathrm{~Hz}, 2 \mathrm{sec}$ stimulus trains showed a nonlinear dependence on stimulus intensity, reaching a maximum $\sim 70 \mathrm{~V}$ but disappearing at $100 \mathrm{~V}$. In contrast, F and DD were lengthened at increasing stimulus intensity, reaching a plateau above 70 V. $B-D$, Induction probabilities of ID, $\mathrm{F}$, and $\mathrm{DD}$ at $50 \mathrm{~V}$ with varying stimulus train durations. Probabilities of induction were determined by the fraction of flies displaying ID, F, and DD. The range of the sample size is indicated, with each fly subjected to only a single trial at each stimulus duration. Maximal ID induction was shifted toward lower stimulus intensities in the mutant flies $(B)$. Single-mutant bss and doublemutant bas $^{1}$ bss $^{1}$ flies exhibited greatly enhanced sensitivity for $\mathrm{F}$ induction $(C)$ but only mild alterations in $\mathrm{DD}$ induction probability $(D)$. Note that induction curves for ID, F, and DD displayed different profiles within each genotype, suggesting involvement of distinct neural circuits.
A
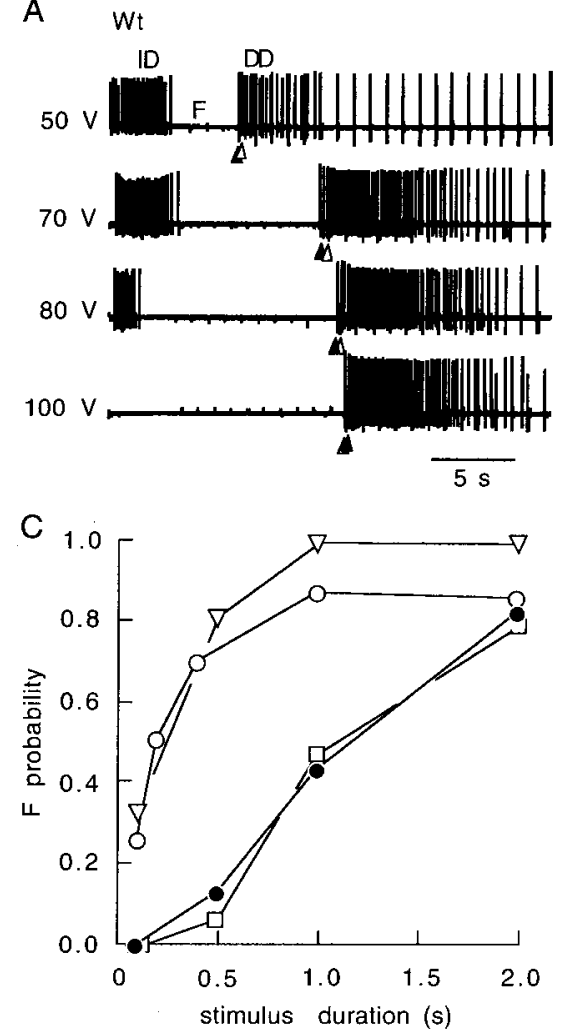
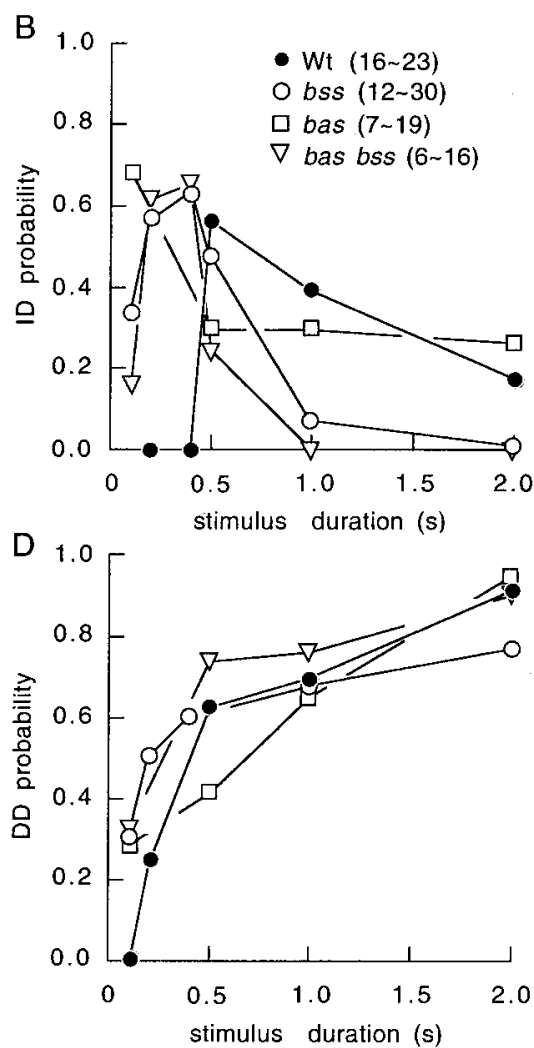
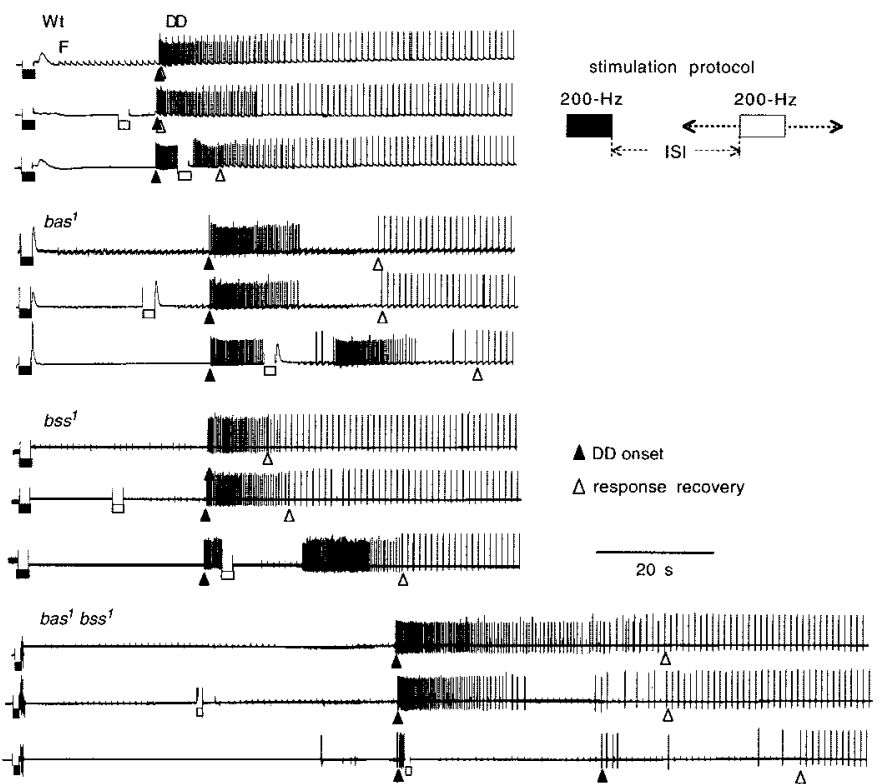

Figure 7. Stereotyped action patterns in the DLM physiological repertoire of wild-type, $b a s^{1}, b s s^{1}$, and $b a s^{1} b s s^{1}$ flies. A DLM repertoire once initiated completed the sequence even when interrupted by a second stimulus. Two identical suprathreshold stimuli $(200 \mathrm{~Hz}, 2 \mathrm{sec}$, except for bas $^{1} b s s^{1}$, which was $1 \mathrm{sec}$; note that ID was suppressed by the suprathreshold stimuli) were delivered at different ISIs. A second stimulus (open rectangle) delivered before the onset of DD produced no change in the DLM repertoire initiated by the first ( filled rectangle), whereas deliveries after the onset of DD introduced a brief suppression of DD and a slight delay in response recovery (compare second and third traces with control in the top trace in each panel). Traces shown are sequential recordings from the same fly with at least $10 \mathrm{~min}$ rests between trials. interposed by intervals longer than those in Figure 7 (minutes vs tens of seconds) were delivered to the head (Fig. 8) (note the suprathreshold suppression of ID in control trials; compare Fig. $6)$. The responses to the second stimuli were analyzed for indications of a refractory period during which $F$ and DD were either modified (because of relative refractoriness) or missing (because of absolute refractoriness). As expected, parameters such as F duration and DD onset time were gradually restored to the characteristic values of each genotype at an increasing interstimulus interval (ISI). For each genotype, traces from the same fly are shown in Figure $8 A$, and pooled data from five flies are plotted for F duration and DD onset time in Figure $8, B$ and $C$. The two stimuli could induce identical responses only if a prolonged ISI (e.g., $10 \mathrm{~min}$ ) was allowed. During the relative refractory period, F duration was shortened, and DD onset occurred earlier with diminished discharge duration seen in Figure $8 A$. Conceptually, the records shown in Figure 7 can be interpreted as the absolute refractory period because the second stimulus lacked any effect when it was delivered before the onset of DD (Fig. 7, middle traces of all genotypes).

Several features about refractory period should be noted. First, it is known that minor disturbances such as rough handling of the culture vial without inducing any seizure-paralysis can still suppress the bang-sensitive behavior. Correspondingly, stimuli insufficient to produce a seizure response could still prevent the full expression of the physiological repertoire (data not shown) (cf. Lee, 2000). Second, within the relative refractory period, modified responses resembled those evoked by near-threshold stimuli (less than optimal expression), suggesting a temporarily increased threshold level. The typical near-threshold responses was evidenced in the $1^{\prime}$ and $3^{\prime}$ traces for wild type and bas (Fig. 8A), showing the reappearance of ID, earlier onset of DD, and shortened $\mathrm{F}$ and DD durations (compare Fig. $6 A$ ). Third, the refrac- 

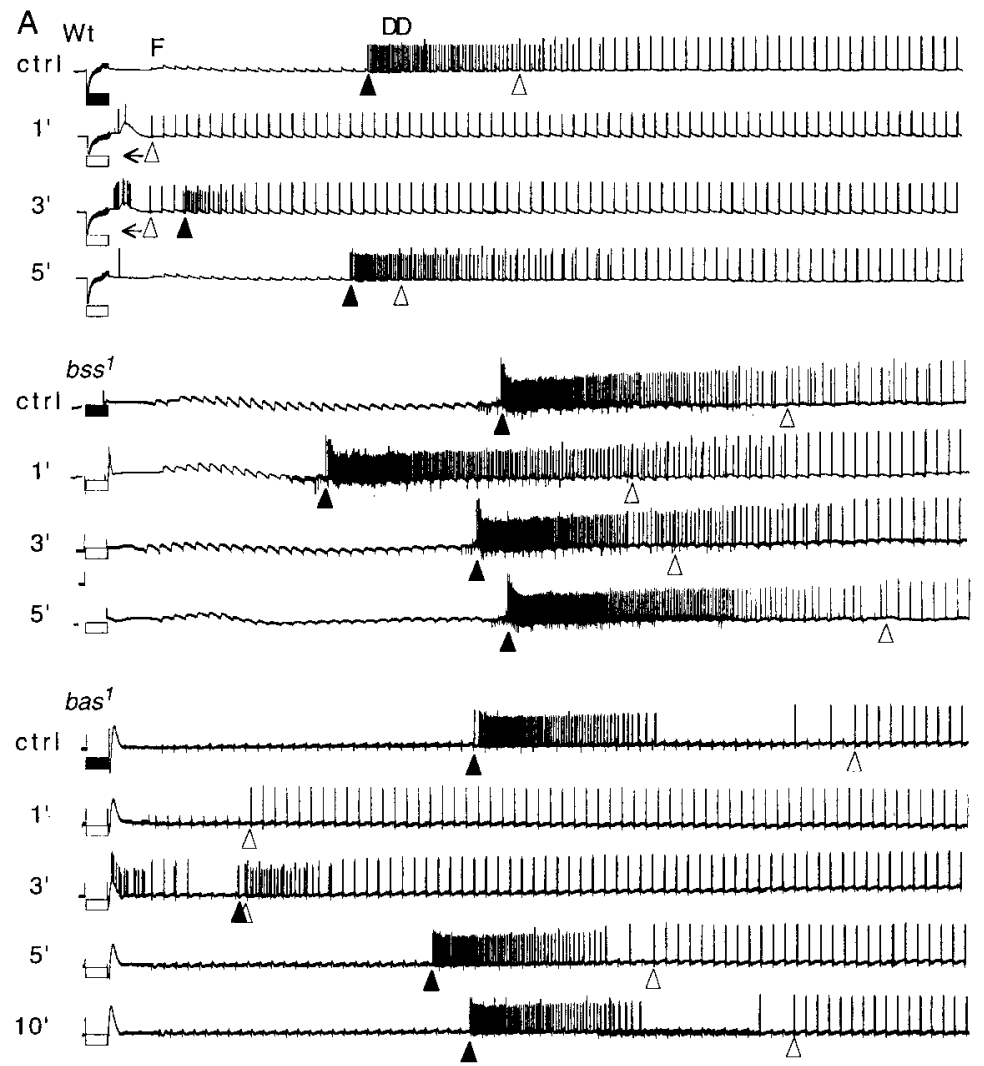
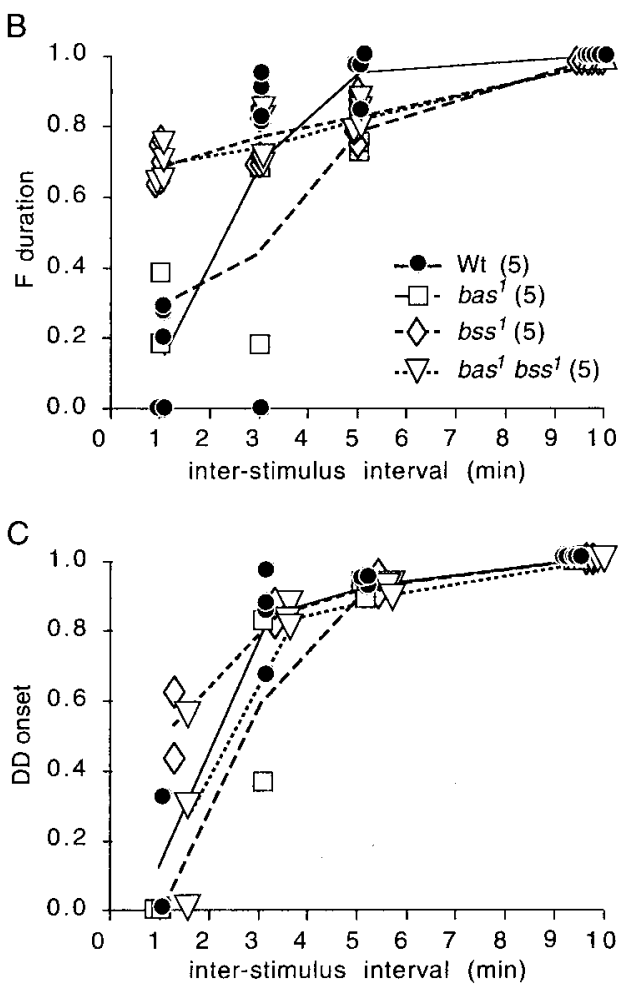

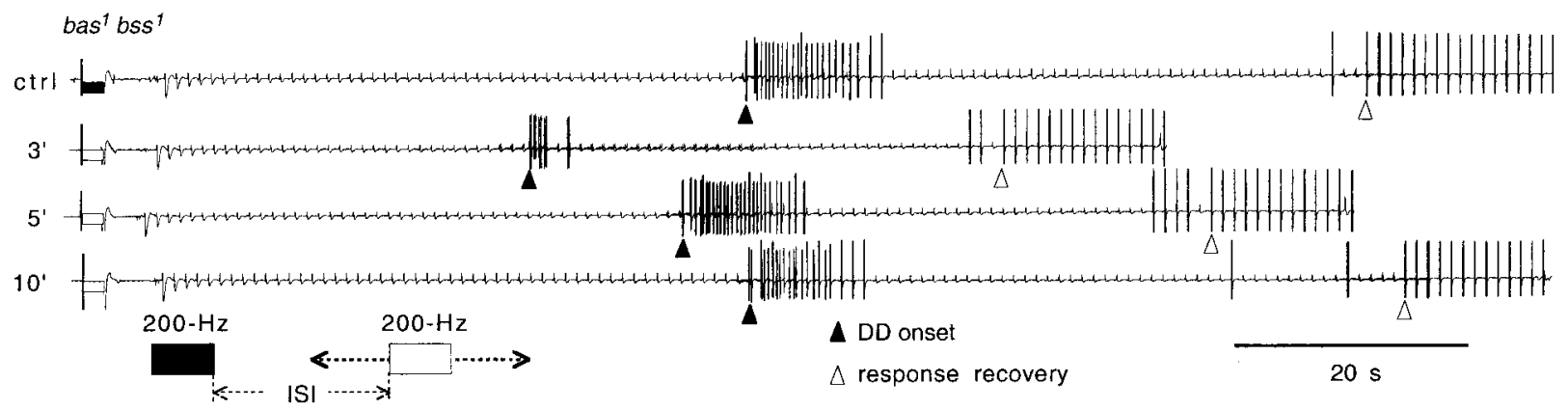

Figure 8. Refractoriness of the DLM repertoire in wild-type and bang-sensitive mutant flies. $A$, Refractoriness indicated by reduced effectiveness of the second electroconvulsive stimulus. F and DD in the responses to the second stimuli were either modified because of relative refractoriness or missing because of absolute refractoriness. In each paired-stimulation trial, the first stimulus $(200 \mathrm{~Hz}, 2 \mathrm{sec}$; filled rectangle) was delivered to a fly rested for at least $10 \mathrm{~min}$, and the second stimulus (open rectangle) followed at different ISIs. In the sequential pair-stimulus trials (at 1, 3, 5, or 10 min ISI, executed in a reversal order in the same fly), the top trace for each genotype (Wt, bas ${ }^{1}, b_{s s}{ }^{1}$, and bas $^{1} b_{s s}{ }^{1}$ ) represents control (ctrl) taken from one of the first responses in the pairs. Note the reappearance of ID in Wt and bas $^{l}$ traces during the relative refractory period, which was suppressed by the suprathreshold stimuli in control traces. $B, C$, Restoration of response during refractory periods as indicated by recovery of $\mathrm{F}$ duration $(B)$ and DD onset time $(C)$, determined in the number of flies indicated. At each ISI, recovery is normalized to control values with line segments connecting the average values for each genotype.

tory period was clearly genotype dependent, with the longest in wild type and shortest in bss and bas bss. Furthermore, the time courses of regaining GF pathway function and DD pattern generation could be modified differentially by mutations. For example, a more gradual restoration of DD duration could be seen in wild-type, $b a s^{1}$, and $b a s^{1} b s s^{1}$ flies in contrast to a rapid and abrupt restoration of full DD expression in $b s s^{1}$ (Fig. 8A).

\section{Candidate circuits involved in DLM electroconvulsive discharges and their anatomical locations}

Although electroconvulsive seizure was more extreme in bangsensitive mutants, wild-type flies expressed the same seizure repertoire at an increased stimulus intensity. Therefore, it is reasonable to hypothesize that the electroconvulsive discharges described above were produced by the circuits that generate normal DLM motor patterns. We examined the various motor activities detectable in DLMs and their modification by electroconvulsion and other experimental conditions. By varying stimulating-recording sites and performing surgical manipulations, we separated anatomically the neural elements responsible for each component of the DLM repertoire.

Relations of ID and DD to other motor patterns in DLMs In tethered flies, both sporadic irregular and sustained regular motor patterns were observed in DLMs under normal conditions 


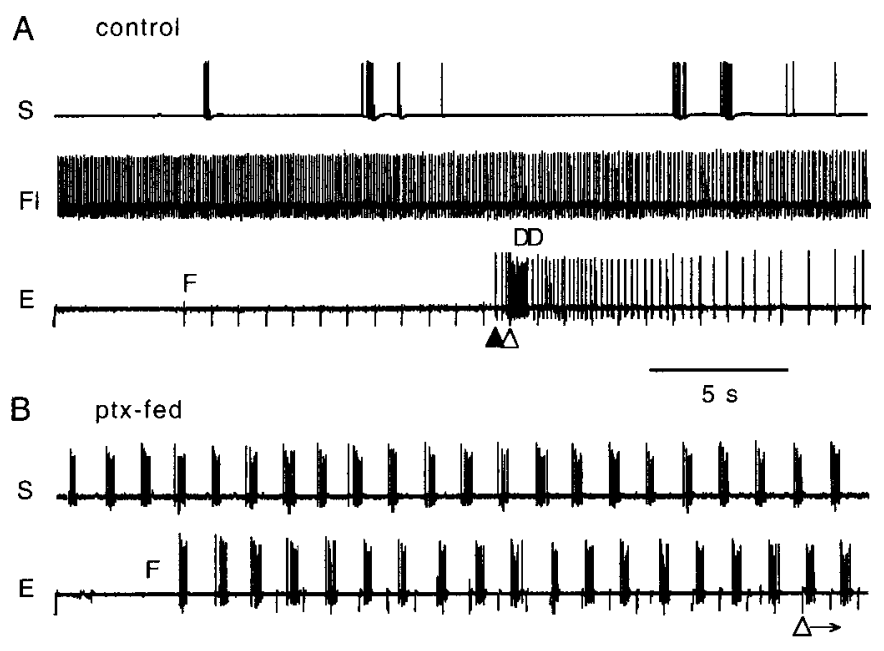

Figure 9. Effects of GABAergic blockade on spontaneous activity and electroconvulsive repertoire of DLMs. Wild-type flies were fed with $1 \mathrm{~mm}$ picrotoxin $(p t x-f e d)$ in food dye-colored medium. $A$, DLMs of control flies (fed with medium without drug) could express three distinct activities: sporadic spontaneous bursts $(S)$ during the rest period, sustained flight activity $(F l)$, and the electroconvulsive repertoire $(E) . B$, Picrotoxin-fed flies exhibited periodic discharges (S) in the DLM and failure of transmission in the GF pathway before experiencing an electroconvulsive shock (data not shown). The periodic bursts appeared to occlude electroconvulsive induction of ID and DD (compare traces $E$ in $A$ and $B$ ), suggesting that GABAergic blockade induces another seizurelike state. Suprathreshold stimuli $(100 \mathrm{~V}, 200 \mathrm{~Hz}$ for $2 \mathrm{sec})$ were applied immediately before traces $E$ in both $A$ and $B$. An arrow next to the open triangle indicates that no recovery from GF pathway failure $(F)$ was observed throughout the recording.

(Fig. 9A). Visual inspections indicated that flight activity of the tethered flies coincided with the regular spike activity $(8-10 \mathrm{~Hz})$ (Fig. 9A, trace F1), whereas twitching and cleaning motions often correlated with sporadic spike activities (trace $S$ ) in DLMs. These nonelectroconvulsion-associated activities have been encountered in all DLMa-f fibers. Significantly, sporadic spike activities were observed during the electroconvulsive repertoire (sometimes superimposing on the DD pattern), especially at nearthreshold stimulus intensities (data not shown). In contrast, flight activities have never been seen during the electroconvulsive repertoire. Furthermore, after an electroconvulsive seizure, flight initiation (either spontaneous or air-puff induced) was suppressed during refractory period, and its reappearance coincided with the regained expression of DD. Therefore, a flight pattern generator could participate in driving the DD pattern, whereas the circuits responsible for sporadic activities appeared to function independently of DD generation.

In mammalian seizure studies, high-frequency electrical stimulus trains have been shown to evoke a stereotypic firing sequence in normal hippocampal slices, reminiscent of the electroconvulsive repertoire seen in Drosophila DLMs, whereas inhibition of GABAergic transmission induces sustained rhythmic bursts (Hablitz, 1984; Stasheff et al., 1985). We therefore examined the possibility that GABAergic blockade in Drosophila could lead to a new pattern of seizure activity. Indeed, a rhythmic bursting pattern distinct from flight and electroconvulsive discharges occurred spontaneously in wild-type flies fed with picrotoxin $(2.0 \mathrm{~mm}$ ), which blocks GABAergic transmission (Fig. 9B, trace $S$, pix-fed). Such conspicuous periodic bursting activity lasted for tens of minutes. In contrast, the sham control fly (fed

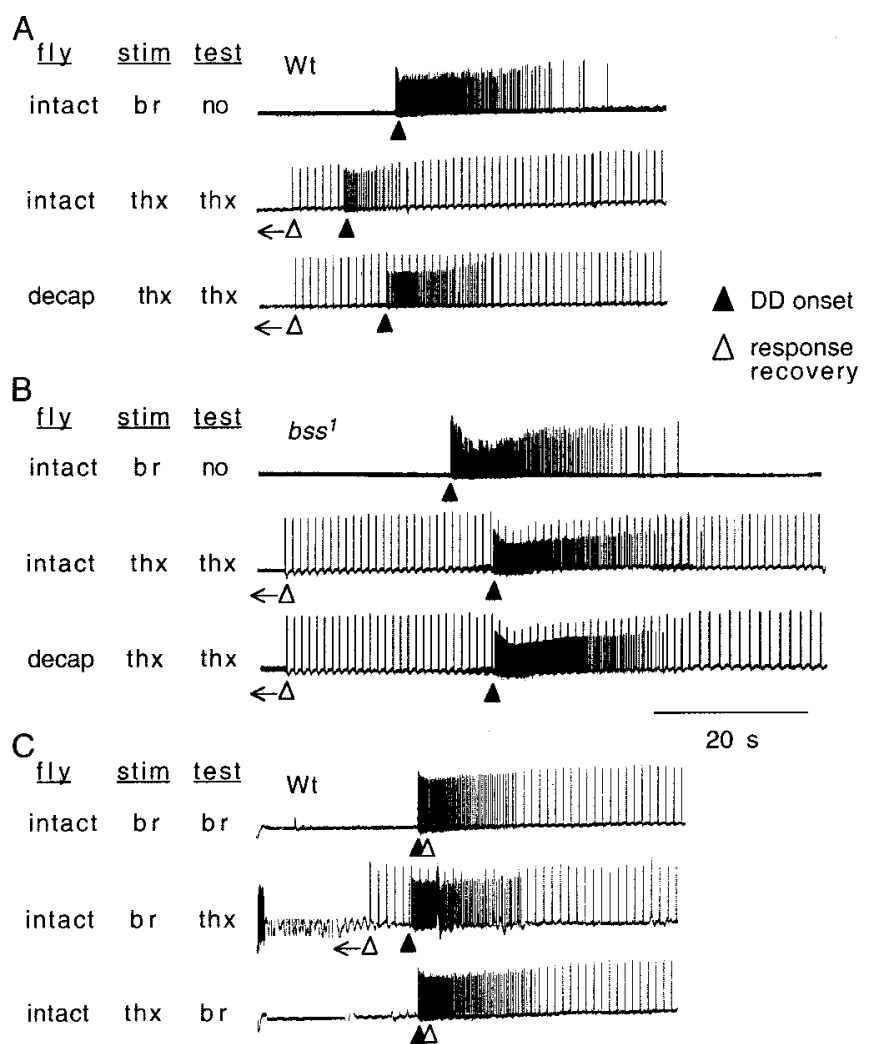

Figure 10. Localization of DD pattern generation to the thorax and $\mathrm{F}$ induction along the GF pathway. $A, B$, Intact and decapitated (decap) preparations were examined for the effect of direct thorax (thx) electroconvulsion (second and third traces) bypassing the brain, as compared with the standard brain $(b r)$ electroconvulsion (top traces) in wild-type $(A)$ and $b s s^{1}$ mutant (B) flies. Thorax electroconvulsive stimulation $(200 \mathrm{~Hz}, 2 \mathrm{sec}$, suprathreshold) could induce characteristic DD discharges of the DLM repertoire with or without the head, suggesting the thoracic localization of DD pattern generator(s). Test pulses $(1 \mathrm{~Hz})$ applied to the thorax after thorax electroconvulsion detected no occurrence of F in DLMs, indicating direct activation of motor neurons by test pulses. $C$, Both brain and thorax electroconvulsion induced failure in DLM response to test pulses delivered to the brain ( first and third traces), but not to the thorax (second trace), suggesting multiple sites along the GF-PSI pathway, but excluding the motor neurons, as the sites of transmission failure. Open triangles with backward-directed arrows indicate the lapse of manual switching time before the onset of test pulses.

with dye-colored food without drug) showed only spontaneous sporadic activity (Fig. 9A, trace $S$ ) or sustained flight patterns (trace $F l$ ) in DLMs. Significantly, in picrotoxin-fed wild-type flies, the characteristic electroconvulsive repertoire was no longer attainable, presumably revealing a different seizure state in which GF failure persisted and induction of ID and DD were occluded (note sustained response failure and absence of ID and DD in Fig. 9B, trace E). In fact, GABAergic blockade caused transmission failure of the GF pathway before the application of electroconvulsion (data not shown). Importantly, picrotoxin feeding in wild-type flies also occluded all other motor patterns, including flight activities (Fig. 9B).

\section{Localization of DD pattern generation in the thorax}

Individual DLM motor neurons and central motor pattern generators for flight have been localized in the thoracic ganglia (King and Wyman, 1980; Ikeda and Koenig, 1988). We examined whether neural circuits in the thorax are sufficient to generate the 

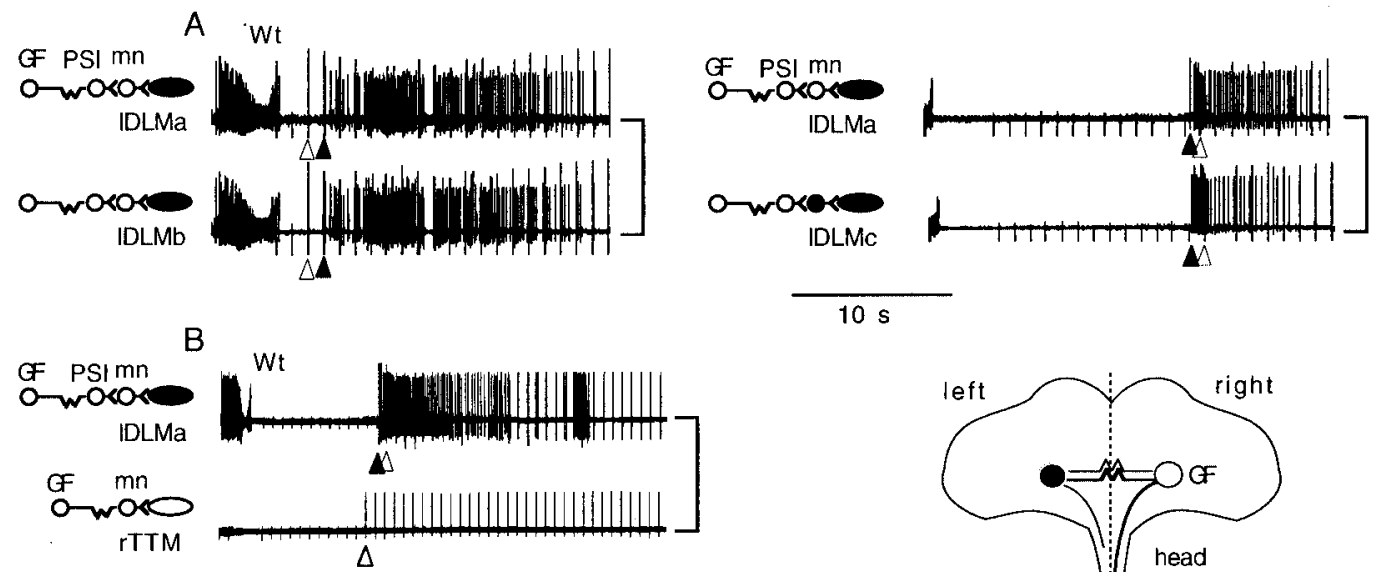

$10 \mathrm{~s}$
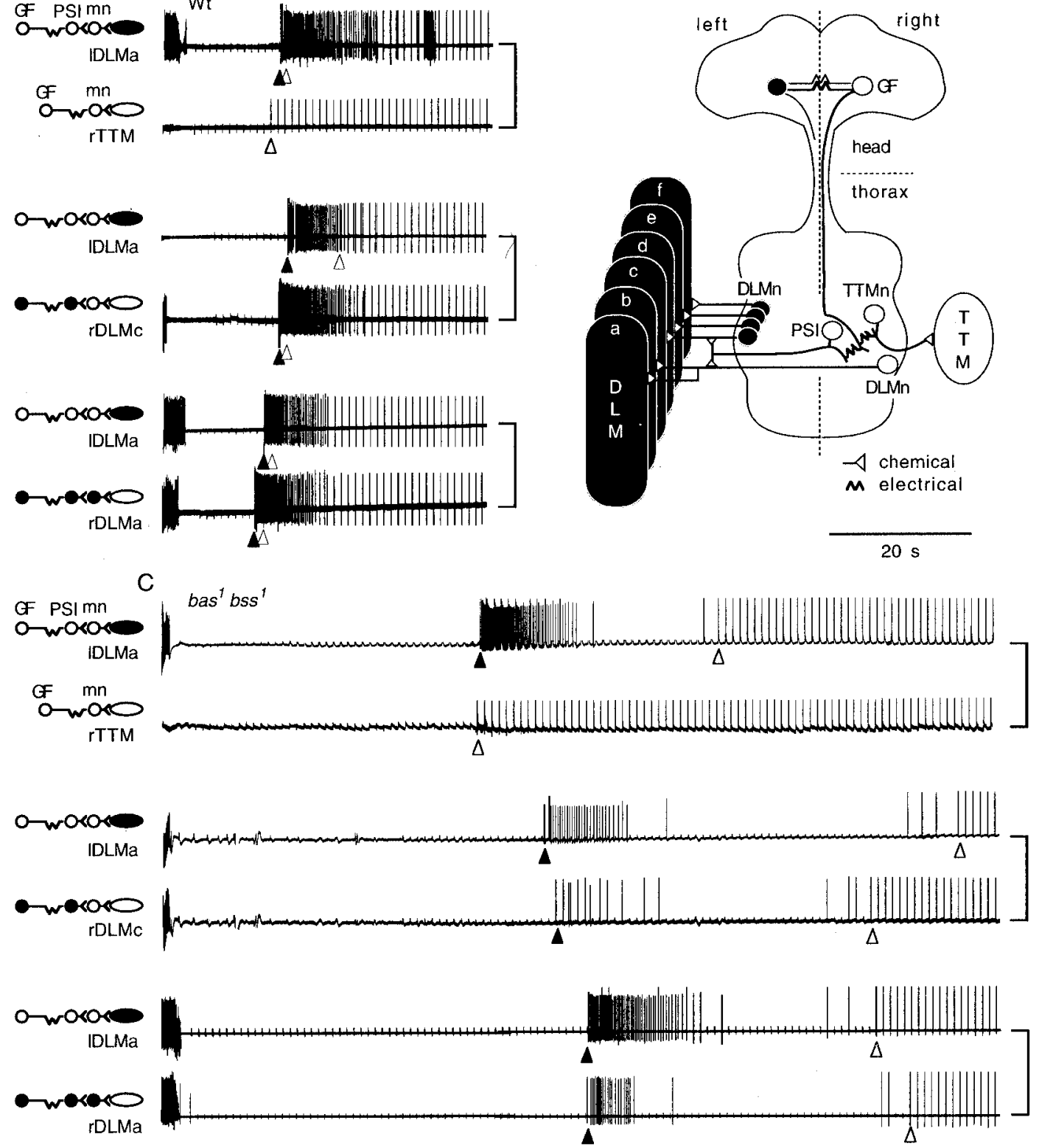

Figure 11. Electroconvulsive repertoires in different motor outputs of the GF pathway in wild-type and bas ${ }^{1}$ bss $^{1}$ double-mutant flies. The inset shows a schematic diagram of one half of the bilaterally symmetrical GF pathway (see Materials and Methods). Cells with somata on the left ( $l$ ) side are filled, and those on the right $(r)$ are open. Isomorphic representations of the GF pathway shown next to individual traces indicate the left- or right-side locations of the neuronal somata and muscle fibers involved. $A$, Discharges in DLM pairs driven by the same versus different motor neurons. A pair of DLM fibers $(I D L M a$ and $l D L M b)$ that share input from the same motor neuron showed synchronized spike firing (left panel). In contrast, firing in IDLMa and IDLMc, which are driven by separate motor neurons, lacked synchronicity despite their similar temporal characteristics of ID, DD, and R (right panel). This indicates that motor neuron activity rather than muscle excitability determines the electroconvulsive spike discharge patterns. $B, C$, Electroconvulsive responses of bilateral muscle pairs in wild-type $(B)$ and bas bss double-mutant $(C)$ flies. Both ID and DD were missing in TTM responses, indicating specificity of the ID and DD pattern generators for DLMs. TTM recovery was faster than that of DLMs, demonstrating a delay imposed by the mutant PSI (compare IDLMa-rTTM in C). Note also the temporal coupling between TTM recovery and DLM DD onset (lDLMa-rTTM pairs), coinciding with the resumption of general CNS activities (compare Fig. 5). Left and right pairs of DLM fibers (IDLM and $r D L M)$, although receiving input from two bilateral GF-PSI chains, showed similar temporal sequence of ID, DD, and R in both wild-type $(B)$ and mutant $(C)$ flies (simultaneous recordings, each pair from a different fly). 
electroconvulsive repertoires in the DLMs. Figure 10 shows the pattern of DLM response to direct thorax stimulation in intact or decapitated flies, supporting the thoracic localization of the circuits involved in DD pattern generation. This was achieved by applying electroconvulsive stimuli across two electrodes positioned bilaterally in the anterior portion of the thorax, which effectively initiated the DD. Furthermore, similar results were obtained from both decapitated wild-type and bss flies to remove the influence from the head (Fig. 10A,B). Electroconvulsive stimuli delivered to the thorax also evoked ID (data not shown). In Figure 10, ID was suppressed by the suprathreshold stimuli used to initiate full-blown DD and F.

Note that after electroconvulsive stimulation applied to either the thorax (Fig. 10A, B, bottom two traces) or the brain (Fig. 10C, middle trace), DLM responses to $1 \mathrm{~Hz}$ test pulses to the thorax persisted (i.e., no failure), presumably because of direct activation of DLM motor neurons. This is in contrast to the standard brain electroconvulsive protocol (Figs. 3-9) in which test pulses were applied to the brain and the DLM response would disappear during GF pathway transmission failure. Moreover, test pulses delivered to the brain after thoracic electroconvulsive stimulation (Fig. 10C, bottom trace) failed to initiate a DLM response, indistinguishable from brain electroconvulsive stimulation (Fig. 10C, compare top and bottom traces). Therefore, either type of electroconvulsive stimuli induced DLM response failure to brain test pulses but no failure to thorax test pulses that could activate DLM motoneurons directly. Thus, severe local shocks to either the brain or the thorax portion of the GF pathway can induce temporary transmission failure.

\section{Contributions of individual circuit elements to the electroconvulsive repertoire}

The well defined GF pathway enabled a systematic analysis of contributions from individual circuit elements to the various components of the electroconvulsive repertoire, ID, DD, and F. Simultaneous recordings from pairs of DLMs and TTMs provided information about temporal correlations among motor output patterns and thus the roles of muscle fibers, motor neurons, and interneurons, as well as putative motor pattern generator circuits outside the GF pathways.

\section{Temporal correlation among different motor outputs of the GF pathway}

Figure 11, inset, shows the placement of the identified nerve and muscle cells innervated by the right cervical GF neuron (for details, see also Fig. $1 A$ and Materials and Methods). For clarity, the symmetrical elements associated with the left GF neuron are omitted. Isomorphic representations shown next to individual traces indicate the left- or right-side (filled or open symbols) locations of the neuronal somata $(G F, P S I, m n)$ and muscle fibers (DLMa-DLMc, TTM) in the GF pathway.

We first examined the possibility that altered muscle excitability, rather than neuronal activity, is responsible for the characteristic DLM discharge pattern in each mutant. We took advantage of the fact that DLMa and DLMb are innervated by the same motor neuron (Fig. 11, inset) and compared their spike activities in simultaneous recordings. We found exact spike-tospike correspondence between the firing patterns recorded in these two muscle fibers (Fig. 11A, left panel), indicating that the electroconvulsive spike pattern is determined by input to the motor neuron. A contrasting result was obtained from simultaneous recording, on the same side, of DLMa and DLMc, which are innervated by separate motor neurons (Fig. 11, inset). In these two muscles, a spike-to-spike correspondence was no longer observed, despite a similar DD onset time (Fig. 11 $A$, right panel), reflecting some degree of independence among motor neurons in expressing electroconvulsive activities. The above finding suggests that fine-tuning in DD patterning involves contributions from individual motor neurons and potentially inputs from different interneurons.

To reveal electroconvulsive discharge profiles specific to distinct functions, we examined the physiological repertoire exhibited in another GF pathway motor output, the jump muscle TTMs. Figure 11 shows traces of simultaneous recordings from TTM-DLM pairs in both wild-type $(B)$ and $b_{a s}^{1} b_{s s^{1}}$ doublemutant $(C)$ flies. The TTM motor neuron receives direct innervation via an electrical synapse from the GF on the same side (Fig. 11, inset). Bursting activity corresponding to either ID or DD was never observed in TTM after electroconvulsive stimuli applied to the brain (Fig. $11 B, C$ ) or thorax (data not shown). This is consistent with the idea that the ID and DD pattern generation involves circuits regulating only specific sets of motor neurons such as DLMns. However, the recovery of TTM from response failure concurred with the time of DD initiation in DLMs (Fig. 11B,C, top pairs), which coincided with onset of general bursting activities in the CNS (Fig. 5). Note the earlier response recovery for the TTM compared with that of DLMs, most evident in the double mutants $b_{a s}{ }^{1} b s s^{1}$, although the two muscles share the same GF input. A possible explanation is that a partial recovery of GF is sufficient to drive the TTM motor neuron directly by electrical coupling but is not enough to induce DLM responses, which depend on successful activation of the interposed PSI chemical transmission (see also mosaic data below).

A comparison of bilateral DLM motor activities suggested that electroconvulsive discharges in muscles on the opposite sides involve recruitment of two separate motor pattern generators. Simultaneous recordings from right-left DLM-DLM pairs indicated that they were activated to display DD approximately, but not exactly, at the same time (Fig. $11 B, C$ ). Thus, bilaterally located pattern generators may be recruited in a coordinated manner to initiate DD activity in different muscles. However, as mentioned above (Fig. 11A), individual DLMns differ in the exact firing pattern even if their somata are located on the same side (see especially bas $^{1}$ bss $^{1}$ double mutants in Fig. $11 B$, middle pair).

\section{Genetic mosaic analysis of the physiological repertoire}

Gynandromorph mosaics of Drosophila have been used to identify the anatomical loci in the nervous system associated with specific behavioral defects and dissect components in a behavioral repertoire (Ikeda and Kaplan, 1970; Hotta and Benzer, 1972, 1976; Burg, 1987). Gyandromorph mosaics consisting of heterozygous and hemizygous tissues (in our study, bas $^{1}$ bss $^{1} /++/ /$ bas $^{1}$ $b_{s s}{ }^{1} / \mathrm{O}$ ) (Fig. 12, inset) were generated by a loss of the unstable ring-X chromosome in a developing heterozygous female embryo. An earlier loss of the ring-X engenders a larger hemizygous mutant patch in the adult fly, identifiable by cuticular phenotypes of recessive markers plus male features of different body parts (see Materials and Methods). If the ring-X loss occurs during the first mitotic division, exact bilateral mosaicism can be found in some gynandromorph flies (Hotta and Benzer, 1972). Such bilateral mosaics are especially usef ul for distinguishing contributions between specific DLMs and their motor neurons to a particular mutant phenotype by taking advantage of the special organiza- 


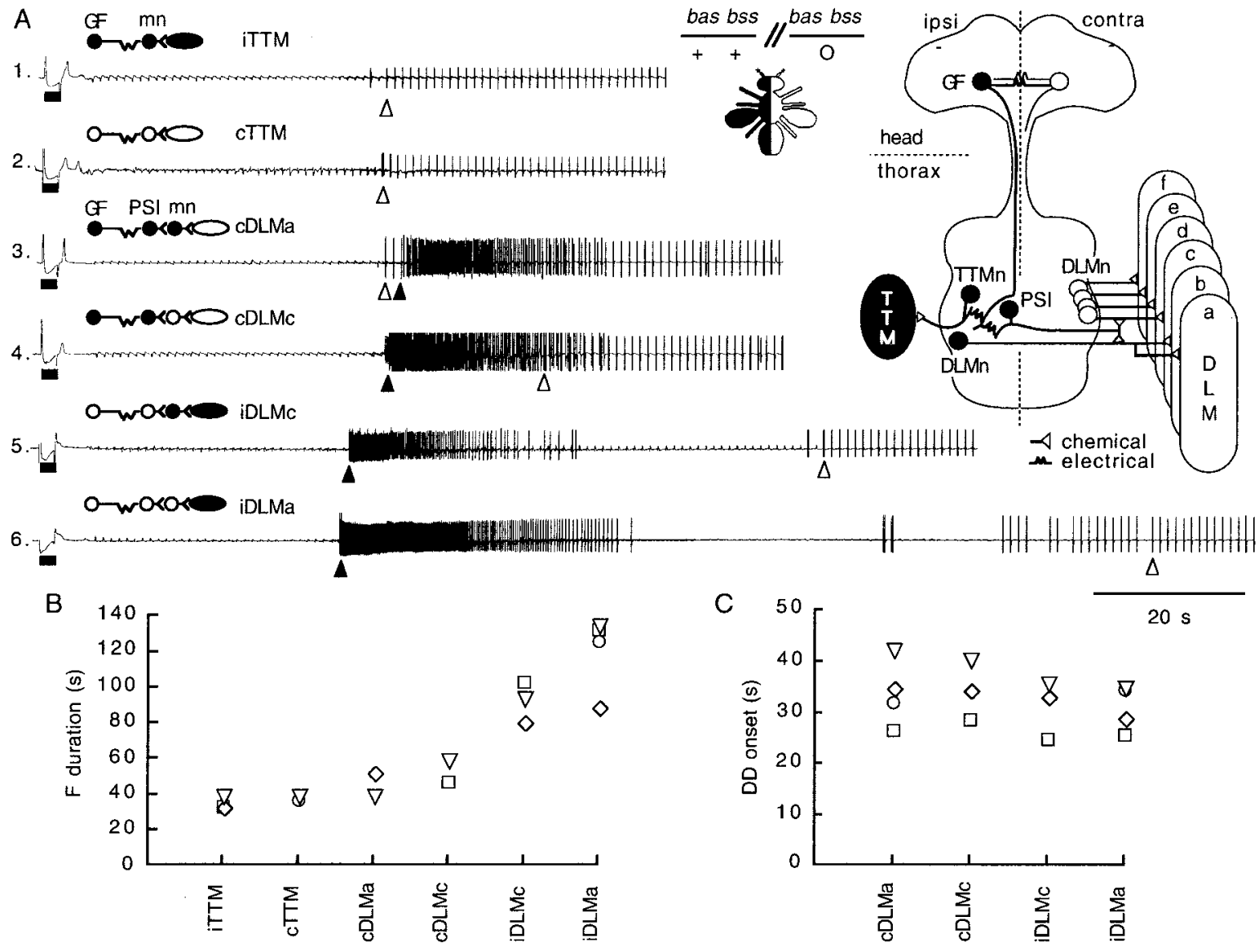

Figure 12. Mutational effects on the identified neurons and muscles of the GF pathway in bas $^{1}$ bss $^{1}$ bilateral mosaic flies. $A$, The inset shows one GF pathway of the bilaterally symmetrical pair (compare Fig. 10, inset) with "control" nerve or muscle elements ( filled) on the left (ipsi) side and "mutant" elements (open) on the right (contra) side. Also shown is a schematic bilateral mosaic with the phenotypically control (bas ${ }^{1}$ bss $\left.^{1} /++\right)$ and mutant $\left(\right.$ bas ${ }^{1}$ $\left.b s s^{1} / \mathrm{O}\right)$ tissue on the left and right, respectively. The predicted genotypes of GF and PSI, and DLM, TTM, and their motor neurons (mn) are determined by whether the soma is located on the ipsilateral $(i)$ control or contralateral $(c)$ mutant side of the body, as shown in the relevant portion of the GF pathway for DLM and TTM inputs next to individual traces. Suprathreshold electroconvulsive stimuli ( filled bars) were applied to compare modifications in the DLM physiological repertoire for DLMs on the mutant side [DLMa (trace 3, cDLMa: no mutant neuron in the pathway) vs DLMc (trace 4, cDLMc: one mutant neuron)] and on the control side [DLMc (trace 5, iDLMc: two mutant neurons) vs DLMa (trace 6, iDLMa: three mutant neurons)]. Note that the number of mutant neurons along the GF pathway correlates with DLM recovery time (open triangle). In contrast, bilateral TTMs showed similar recovery regardless of the number of mutant neurons (trace 1, iTTM: all normal cells; trace 2, cTTM: all mutant cells), suggesting bilateral coupling for the GF pathways. Note also the similar timing of DLM DD onset and TTM response recovery on both sides regardless of the number of mutant elements along the GF pathway (traces 1-6; compare Fig. 10). B, C, Consistent results were obtained from four bas ${ }^{1}$ bss $^{1}$ bilateral mosaics. Separate symbols were used to distinguish data from different mosaic flies. F duration of DLM recovery lengthened with increased numbers of predicted mutant neurons in the GF pathway $(B)$. In contrast, DD onset time was independent of the number of mutant neurons in the GF-PSI-mn chain $(C)$.

tion of the GF pathway (compare Fig. 12, inset). This has been elegantly demonstrated by Koenig and Ikeda (1983) to localize shibire mutant defects to the presynaptic motor neurons. Here we used a similar approach to distinguish the contributions of different circuit elements to the electroconvulsive seizure repertoire in bas $^{1}$ bss $^{1}$ double-mutant mosaics. In a perfectly bilateral mosaic, the genotypes of the GF, PSI, mn, and muscle cells can be assigned as indicated by the isomorphic circuits shown above individual traces in Figure $12 A$. It can be seen that different groups of DLMs are innervated by different numbers of mutant neurons (open symbols) in the GF-PSI-DLMn chain of a bilateral mosaic.

Four bilateral bas $^{1}$ bss $^{1}$ mosaics have been examined, demonstrating consistent results. Example traces obtained from one (Fig. 12A) illustrate how the repertoires of DLMa, DLMc, and TTM fibers ipsilateral $(i)$ or contralateral $(c)$ to the control side were influenced by mutant elements in the GF-PSI-DLMn or
GF-TTMn chains (see corresponding isomorphic chains and inset in Fig. 12A). Traces $3-6$ in Figure $12 A$ demonstrate that the recovery time (open triangles) of the DLM responses to test stimuli applied to the brain was progressively lengthened as the number of presumptive mutant neurons in the GF pathway increased (from zero in trace 3 to three in trace 6). However, when the DLM motor neuron was mutant, the recovery time was delayed, whether the GF-PSI pair was wild type (traces 3 and 4) or mutant (traces 5 and 6 ). In contrast, DD onset time (filled triangles) was clearly less dependent on the number of mutant neurons in the GF pathway (traces 3-6), supporting the notion that the recruited circuits for DD generation in DLMs can operate independent of the GF pathway. Consistent with the results shown in Figure 11, there was no DD-like activity in TTMs (traces 1 and 2), and their recovery time coincided roughly with the onset time of DD in DLMs (traces 3-6). Moreover, TTM response recovery was not significantly influenced by the genotypes of the 
neuronal elements, even if both GF and TTM motor neurons were mutant (compare traces 1 and 2). Interestingly, the recovery time of the bilateral TTM pair in the double-mutant mosaic was intermediate between those seen in wild-type and bas bss doublemutant flies (compare Fig. 12, traces 1 and 2, with Fig. 11B, C, top pairs), reflecting electrical coupling between the two GF neurons (Phelan et al., 1996; Sun and Wyman, 1996).

The above observations were true for all four bilateral $\mathrm{bas}^{1} \mathrm{bss}^{1}$ gyandromorphs, as summarized for F duration (Fig. 12B) and DD onset time (Fig. 12C) of TTM and DLMs with different numbers of mutant neurons in their GF pathway inputs. Note that in each bilateral mosaic, DD onset time was again intermediate between those seen in wild-type and bas bss double-mutant flies (compare Figs. $12 C$ and $4 B$ ). The close correspondence among the DD onset time of bilateral DLMs and the similar recovery time of the bilateral TTM pair again indicate an overall resumption of the CNS activity (Fig. 5), regardless of the locations on the mutant or control side. These results based on as $^{1}$ bss $^{1}$ bilateral mosaics were further supported by observations in 5 additional bilateral eas ${ }^{2}$ bas $^{1}$ double-mutant gyandromorphs (data not shown) and 12 bas $^{1}$ bss $^{1}$ mosaic flies with bilateral divisions in either head or thorax (Lee, 2000).

\section{DISCUSSION}

\section{Physiological correlates of the bang-sensitive behavior}

We explored the electrophysiological correlates of the electroconvulsive behavioral repertoire evoked in tethered flies, which parallels aspects of the seizure behavior in unrestrained bangsensitive mutant flies. Pavlidis and Tanouye (1995) first demonstrated a DLM spike discharge in the GF pathway of bang-sensitive mutants after high-frequency $(200-\mathrm{Hz})$ brain stimulation. This spike discharge shows an onset time and pattern corresponding to ID. With the additional characterization of DD, response recovery, and refractory period in the current study (cf. Lee and Wu, 1998,1 2000; Lee, 2000), the physiological repertoire described here correlates with the complete sequence of electroconvulsive seizure behavior, as confirmed in simultaneous video recordings. It should be noted that the duration of individual pulses within the $200 \mathrm{~Hz}$ electroconvulsive stimulus was much briefer in the present study compared with that applied in previous reports ( $0.1 \mathrm{vs} 0.5 \mathrm{msec}$ ) (Pavlidis and Tanouye, 1995; Kuebler and Tanouye, 2000), resulting in higher threshold voltages $(\sim 40$ vs $\sim 10 \mathrm{~V})$. Although the total current flow is similar for both cases, the present protocol offers a wider range of voltage control to fine tune the physiological responses in different mutants (Figs. $4,6)$. This physiological repertoire is very robust and reproducible, because nearly identical DD onsets and durations as well as F durations were obtained during repetitive trials (up to 40 times) from a wild-type fly. This enables quantitative analysis of seizure parameters using Drosophila mutants and specific drugs to uncover cellular mechanisms underlying seizure and bang-sensitive behavior (Lee and $\mathrm{Wu}, 1998,2000$ ).

A stereotyped action pattern and refractory period are the prominent features in electroconvulsive responses of tethered flies as well as in the seizure behavior of unrestrained bangsensitive mutants. Similar phenomena have been observed in vertebrate seizures (Nutt et al., 1981; Fisher, 1989), including those occurring in electroconvulsive therapy (Sackeim, 1987) and epileptic conditions (Quesney, 1986; Williamson and Spencer, 1986) in humans. On overwhelming challenges (electroconvulsion) or under pathological conditions (epilepsy), individual motor programs for normal function (Prince, 1999) can be recruited to express a stereotyped action sequence. Neuroethological studies have illustrated some neural organization principles underlying fixed action patterns that are essential for the survival and propagation of the species, such as feeding (Kater, 1974; HarrisWarrick and Marder, 1991), crawling (Johnston and Levine, 1996), flight (Levine and Wyman, 1973; Koenig and Ikeda, 1980), and escape (Zucker et al., 1971; Trimarchi and Schneiderman, 1993). Studies of altered components in the seizure repertoire using defined Drosophila mutations may thus provide further molecular and cellular insights into network hierarchy and interaction principles common to fixed-action patterns and seizure across phyla.

\section{Circuit organization for the physiological repertoire}

Our results suggest that the stereotypic activity pattern in seizure of bang-sensitive flies involves a large portion of the nervous system (Fig. 5). Therefore, the repertoire reflects the sequential activation of different circuits, each distinct in physiological properties and anatomical locations as demonstrated by simultaneous recordings at different sites, surgical manipulation, and mosaic analysis (Figs. 10-12). Furthermore, circuit activities associated with individual components of the seizure repertoire could be selectively modulated by varying stimulus intensity, especially near the threshold level (Fig. 6) and during the relative refractory period (Fig. 8).

Activities from the left and right sides of the GF outputs appear to be closely associated, because both sides of the bilateral mosaics display similar thresholds and DD onset times of DLMs (Fig. 12), as well as nearly identical recovery times of the TTMs (Fig. 12). The ID and DD spike discharges of DLMs reflect circuit activities independent of the GF-PSI pathway, as evidenced by their occurrence during GF failure (Fig. 11C). Furthermore, the marked differences in their threshold profiles and firing patterns (Fig. 6) raise the possibility that the generation of ID and DD may involve two distinct sets of circuits. ID discharge occurs only at near threshold intensities but becomes suppressed at higher intensities, potentially involving both excitatory and inhibitory inputs triggered at different stimulus levels. In contrast, DD has a higher threshold and shows little variation in spike frequency profile and burst duration above a suprathreshold level. However, the gating of both ID and DD may involve a single network, with ID produced during the upslope and DD during the downslope of its activity.

The DLM discharge patterns display considerable plasticity modifiable by various factors, including stimulus intensity, previous activity, and mutational perturbation (Figs. 4, 6-8) (cf. Lee, 2000). Our results are consistent with the idea that these stereotyped DLM activities are derived from neural circuits subserving normal function. Specifically, a flight pattern generator most likely participates in DD pattern generation, with possible contributions from individual motor neurons and associated interneurons, as supported by the following lines of evidence. (1) As in flight, DD expression did not rely on transmission via the giant fiber pathway (Fig. 11C) (Trimarchi and Scheiderman, 1995). (2) Only flight muscles DLMs, but not jump muscles TTMs, expressed DD activities. (3) Similar to the case of flight (Levine and Wyman, 1973; Koenig and Ikeda, 1980), DD spike activities of different DLMs were not synchronized and did not display strict phase locking. Individual muscles exhibited considerable independence in the timing of spikes, despite the fact that they follow a general profile of firing pattern (Fig. 11). (4) DLM flight and DD activities were mutually exclusive. We never observed flight 
during the electroconvulsive response repertoire. (5) There was a period of flight suppression after electroconvulsion, which coincided with the refractory period of DD. Moreover, both flight and DD were occluded at the same time by a sustained, periodic bursting activities induced by picrotoxin feeding (Fig. 9).

Complex interactions among circuit components to generate DD pattern is also indicated by the $b^{1} s^{1} b s s^{1}$ double-mutant phenotype, which is not a simple superposition of the single mutant phenotypes (Figs. 4, 11). The interaction between bas ${ }^{1}$ and $b s s^{1}$ unexpectedly reduces DD duration to nearly the control level (Fig. 4A,B), despite the fact that seizure susceptibility, DD onset time, and $\mathrm{F}$ duration becomes much more extreme in the double mutant (Figs. 4, 6, 11).

In bilateral bas $^{1}$ bss ${ }^{1}$ mosaics, the well defined GF pathway makes it possible to trace the contributions to the modified repertoire from individual mutant neurons (Fig. 12). The phenomenon of a synchronized rebound of global activities after a period of suppression is best illustrated by the nearly identical timing of DD onset in DLMs and recovery of TTMs (Fig. 11), regardless of the neuronal genotypes within the circuits (Fig. 12) (data not shown). In contrast to TTM recovery, there is a clear dependence of DLM recovery on the number of mutant elements in the GF-PSI-DLMn chain (Fig. 12A, compare traces 1-2 and 3-6). This likely reflects the possibility that direct activation of TTMn by the GF via gap junctions (Tanouye and Wyman, 1980) is more robust than DLMn activation mediated by PSI via chemical transmission (Gorczyca and Hall, 1984). When DLMns were directly activated by thorax stimulation to bypass PSI, no failure of DLM response was seen after electroconvulsive stimulation (Fig. 10).

\section{Genetic heterogeneity of the bang-sensitive seizure behavior}

Vertebrate seizure and human epilepsy are known to have a highly heterogeneous etiology involving many categories of genes. Studies on epileptic seizure in human and animal models have implicated defects in $\mathrm{Na}^{+}, \mathrm{Ca}^{2+}$, and $\mathrm{K}^{+}$channels, CaM Kinase II, and nonreceptor tyrosine kinases (Puranam and McNamara, 1999). Similarly, molecular defects of bang-sensitive mutants in Drosophila appear to involve diverse categories of proteins. In the few cases of gene products identified so far, no immediate functional link is evident among them. These include ethanolamine kinase (Pavlidis et al., 1994) and a mitochondrial ribosomal protein (tko) (Royden et al., 1987).

In previous studies, hyperexcitability of motor axon terminals has been demonstrated in bss larval neuromuscular preparations (Jan and Jan, 1978; Ganetzky and Wu, 1982). In addition, $\mathrm{Na}^{+} / \mathrm{K}^{+}$pump defects in the Drosophila mutant 2206 have been reported to cause a bang-sensitive-like phenotype (Schubiger et al., 1994). Therefore, conditions of hyperkalemia caused by defective $\mathrm{Na}^{+} / \mathrm{K}^{+}$pump may lead to hyperexcitability and bang sensitivity. Examination of the $\mathrm{Na}^{+} / \mathrm{K}^{+}$pump $\alpha$-subunit mutant 2206 indeed indicated somewhat reduced seizure threshold and extended GF failure and DD durations (data not shown), despite the absence of the typical bang-sensitive repertoire (compare Fig. 2) during mechanical stress. Significantly, defective membrane repolarization processes in $\mathrm{K}^{+}$channel mutants did not significantly enhance sensitivity to either mechanical or electrical shocks (Lee and Wu, 1998). Therefore, a number of interacting subcellular mechanisms may be required to reach a critical level of seizure susceptibility to express the characteristic bangsensitive behavior.
A clearer picture of the involved molecular networks may emerge once additional gene products of available bang-sensitive mutants are identified. New mutant screens will undoubtedly enlarge the list of bang sensitivity-related genes. In addition, this forward genetic approach may be complemented by investigation of other categories of Drosophila mutations of well defined mechanisms to determine their influence on seizure.

\section{REFERENCES}

Baird DH, Koto M, Wyman RJ (1993) Dendritic reduction in Passover, a Drosophila mutant with a defective giant fiber neuronal pathway. J Neurobiol 24:971-984.

Benzer S (1971) From the gene to behavior. JAMA 218:1015-1022.

Blagburn JM, Alexopoulos H, Davies JA, Bacon JP (1999) Null mutation in shaking-B eliminates electrical, but not chemical, synapses in the Drosophila giant fiber system: a structural study. J Comp Neurol 404:449-458.

Burg MG (1987) Genetic and mosaic analysis of mutations which alter nerve and muscle excitability in Drosophila melanogaster: effects on development and behavior. PhD thesis, University of Iowa.

Burg MG, Wu C-F (1987) A class of Drosophila behavioral mutants that are sensitive to both mechanical vibrations and temperature conditions. Soc Neurosci Abstr 13:619.

Engel JE, Wu CF (1992) Interactions of membrane excitability mutations affecting potassium and sodium currents in the flight and giant fiber escape systems of Drosophila. J Comp Physiol [A] 171:93-104.

Engel JE, Wu C-F (1994) Altered mechanoreceptor response in Drosophila bang-sensitive mutants. J Comp Physiol [A] 175:267-278.

Engel JE, Wu CF (1996) Altered habituation of an identified escape circuit in Drosophila memory mutants. J Neurosci 16:3486-3499.

Engel JE, Wu CF (1998) Genetic dissection of functional contributions of specific potassium channel subunits in habituation of an escape circuit in Drosophila. J Neurosci 18:2254-2267.

Fisher RS (1989) Animal models of the epilepsies. Brain Res Brain Res Rev 14:245-278.

Ganetzky B, Wu CF (1982) Indirect suppression involving behavioral mutants with altered nerve excitability in Drosophila melanogaster. Genetics 100:597-614.

Gorczyca M, Hall JC (1984) Identification of a cholinergic synapse in the giant fiber pathway of Drosophila using conditional mutations of acetylcholine synthesis. J Neurogenet 1:289-313.

Grigliatti TA, Hall L, Rosenbluth R, Suzuki DT (1973) Temperaturesensitive mutations in Drosophila melanogaster. XIV. A selection of immobile adults. Mol Gen Genet 120:107-114.

Hablitz JJ (1984) Picrotoxin-induced epileptiform activity in hippocampus: role of endogenous versus synaptic factors. J Neurophysiol 51:1011-1027.

Harris-Warrick RM, Marder E (1991) Modulation of neural networks for behavior. Annu Rev Neurosci 14:39-57.

Hotta Y, Benzer S (1972) Mapping of behaviour in Drosophila mosaics. Nature 240:527-535.

Hotta Y, Benzer S (1976) Courtship in Drosophila mosaics: sex-specific foci for sequential action patterns. Proc Natl Acad Sci USA 73:4154-4158

Ikeda K, Kaplan WD (1970) Unilaterally patterned neural activity of gynandromorphs, mosaic for a neurological mutant of Drosophila melanogaster. Proc Natl Acad Sci USA 67:1480-1487.

Ikeda K, Koenig JH (1988) Morphological identification of the motor neurons innervating the dorsal longitudinal flight muscle of Drosophila melanogaster. J Comp Neurol 273:436-444.

Jan YN, Jan LY (1978) Genetic dissection of short-term and long-term facilitation at the Drosophila neuromuscular junction. Proc Natl Acad Sci USA 75:515-519.

Johnston RM, Levine RB (1996) Crawling motor patterns induced by pilocarpine in isolated larval nerve cords of Manduca sexta. J Neurophysiol 76:3178-3195.

Judd BH, Shen MW, Kaufman TC (1972) The anatomy and function of a segment of the X chromosome of Drosophila melanogaster. Genetics $71: 139-156$.

Kater SB (1974) Feeding in Helisoma trivolvis: the morphological and physiological bases of a fixed action pattern. Am Zoology 14:1017-1036.

King DG, Wyman RJ (1980) Anatomy of the giant fibre pathway in Drosophila. I. Three thoracic components of the pathway. J Neurocytol 9:753-770.

Koenig JH, Ikeda K (1980) Neural interactions controlling timing of flight muscle activity in Drosophila. J Exp Biol 87:121-136.

Koenig JH, Ikeda K (1983) Evidence for a presynaptic blockage of transmission in a temperature-sensitive mutant of Drosophila. J Neurobiol 14:411-419.

Kuebler D, Tanouye MA (2000) Modifications of seizure susceptibility in Drosophila. J Neurophysiol 83:998-1009. 
Kupferberg H (2001) Animal models used in the screening of antiepileptic drugs. Epilepsia 42 [Suppl] 4:7-12.

Lee J (2000) Electroconvulsive responses in neural circuits underlying seizure behavior in Drosophila: electrophysiological and genetic analysis of bang-sensitive, ion channel, and synaptic mutants. PhD thesis, University of Iowa.

Lee J, Wu C-F (1998) Modified excitability and seizure-and-paralysis repertoire in the jump-and-flight escape system of Drosophila: genetic interaction between sodium channel and bang-sensitive mutants. Soc Neurosci Abstr 24:16.

Lee J, Wu C-F (2000) Electroconvulsion-induced seizure repertoire in the jump-and-flight escape system of Drosophila: effects of synaptic transmission modifications and bang-sensitive mutations. Soc Neurosci Abstr 26:221.

Levine JD, Hughes M (1973) Stereotaxic map of the muscle fibers in the indirect flight muscles of Drosophila melanogaster. J Morphol 140:153-158.

Levine JD, Wyman RJ (1973) Neurophysiology of flight in wild-type and a mutant Drosophila. Proc Natl Acad Sci USA 70:1050-1054.

Lindsley DL, Zimm GG (1992) The genome of Drosophila melanogaster. New York: Academic.

McNamara JO (1999) Emerging insights into the genesis of epilepsy. Nature 399:A15-22.

Noebels JL (1996) Targeting epilepsy genes. Neuron 16:241-244.

Nutt DJ, Cowen PJ, Green AR (1981) Studies on the post-ictal rise in seizure threshold. Eur J Pharmacol 71:287-295.

Pavlidis P, Tanouye MA (1995) Seizures and failures in the giant fiber pathway of Drosophila bang-sensitive paralytic mutants. J Neurosci 15:5810-5819.

Pavlidis P, Ramaswami M, Tanouye MA (1994) The Drosophila easily shocked gene: a mutation in a phospholipid synthetic pathway causes seizure, neuronal failure, and paralysis. Cell 79:23-33.

Phelan P, Nakagawa M, Wilkin MB, Moffat KG, O'Kane CJ, Davies JA, Bacon JP (1996) Mutations in shaking-B prevent electrical synapse formation in the Drosophila giant fiber system. J Neurosci 16:1101-1113.

Prince DA (1999) Epileptogenic neurons and circuits. Adv Neurol 79:665-684.

Puranam RS, McNamara JO (1999) Seizure disorders in mutant mice: relevance to human epilepsies. Curr Opin Neurobiol 9:281-287.
Quesney LF (1986) Clinical and EEG features of complex partial seizures of temporal lobe origin. Epilepsia 27:S27-S45.

Reichert H, Wine JJ (1982) Neural mechanisms for serial order in a stereotyped behaviour sequence. Nature 296:86-87.

Royden CS, Pirrotta V, Jan LY (1987) The tko locus, site of a behavioral mutation in Drosophila melanogaster, codes for a protein homologous to prokaryotic ribosomal protein S12. Cell 51:165-173.

Sackeim H (1987) Seizure threshold in electroconvulsive therapy. Arch Gen Psychiatry 44:355-360.

Schubiger M, Feng Y, Fambrough DM, Palka J (1994) A mutation of the Drosophila sodium pump alpha subunit gene results in bang-sensitive paralysis. Neuron 12:373-381.

Stafstrom CE, Tempel BL (2000) Epilepsy genes: the link between molecular dysfunction and pathophysiology. Ment Retard Dev Disabil Res Rev 6:281-292.

Stasheff SF, Bragdon AC, Wilson WA (1985) Induction of epileptiform activity in hippocampal slices by trains of electrical stimuli. Brain Res 344:296-302.

Sun YA, Wyman RJ (1996) Passover eliminates gap junctional communication between neurons of the giant fiber system in Drosophila. J Neurobiol 30:340-348.

Tanouye M, Wyman R (1980) Motor outputs of giant nerve fiber in Drosophila. J Neurophysiol 44:405-421.

Trimarchi J, Schneiderman A (1993) Giant fiber activation of an intrinsic muscle in the mesothoracic leg of Drosophila melanogaster. J Exp Biol 177:149-167.

Trimarchi J, Scheiderman A (1995) Different neural pathways coordinate Drosophila flight initiations evoked by visual and olfactory stimuli. J Exp Biol 198:1099-1104.

Williamson PD, Spencer SS (1986) Clinical and EEG features of complex partial seizures of extratemporal origin. Epilepsia 27:S46-S63.

Wu C-F, Lee J (2001) Electroconvulsive seizure-and-paralysis repertoire in motor systems bang-sensitive, ion-channel, and neurotransmitter mutants of Drosophila. J Neurogenet 15:72.

Wyman RJ, Thomas JB (1983) What genes are needed to make an identified synapse? Cold Spring Harbor Symp Quant Biol 48:641-652.

Zucker RS, Kennedy D, Selverston AI (1971) Neuronal circuit mediating escape responses in crayfish. Science 173:645-650. 